\title{
Renormalization Hopf algebras and combinatorial groups
}

\author{
Alessandra Frabetti \\ Université de Lyon, Université Lyon 1, CNRS, \\ UMR 5208 Institut Camille Jordan, \\ Bâtiment du Doyen Jean Braconnier, \\ 43, blvd du 11 novembre 1918, F-69622 Villeurbanne Cedex, France. \\ frabetti@math.univ-lyon1.fr
}

May 5, 2008

\begin{abstract}
These are the notes of five lectures given at the Summer School Geometric and Topological Methods for Quantum Field Theory, held in Villa de Leyva (Colombia), July 2-20, 2007. The lectures are meant for graduate or almost graduate students in physics or mathematics. They include references, many examples and some exercices. The content is the following.

The first lecture is a short introduction to algebraic and proalgebraic groups, based on some examples of groups of matrices and groups of formal series, and their Hopf algebras of coordinate functions.

The second lecture presents a very condensed review of classical and quantum field theory, from the Lagrangian formalism to the Euler-Lagrange equation and the Dyson-Schwinger equation for Green's functions. It poses the main problem of solving some non-linear differential equations for interacting fields.

In the third lecture we explain the perturbative solution of the previous equations, expanded on Feynman graphs, in the simplest case of the scalar $\phi^{3}$ theory.

The forth lecture introduces the problem of divergent integrals appearing in quantum field theory, the renormalization procedure for the graphs, and how the renormalization affects the Lagrangian and the Green's functions given as perturbative series.

The last lecture presents the Connes-Kreimer Hopf algebra of renormalization for the scalar theory and its associated proalgebraic group of formal series.
\end{abstract}

\section{Contents}

Lecture I - Groups and Hopf algebras 2

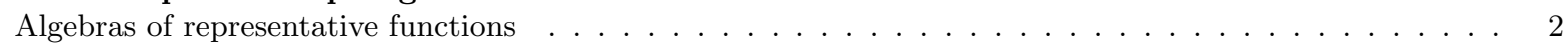

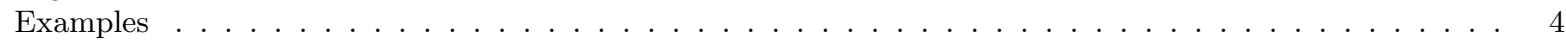

Groups of characters and duality . . . . . . . . . . . . . . . . . . . . . . . 9

Lecture II - Review on field theory 11

4 Review of classical field theory . . . . . . . . . . . . . . . . . . . . . . . 11

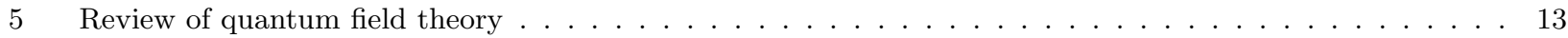

Lecture III - Formal series expanded over Feynman graphs 16

6 Interacting classical fields . . . . . . . . . . . . . . . . . . . . . . . . . . 17

7 Interacting quantum fields . . . . . . . . . . . . . . . . . . . . . . . . . . 18

8 Field theory on the momentum space . . . . . . . . . . . . . . . . . . . . 20

Lecture IV - Renormalization 23

9 Renormalization of Feynman amplitudes . . . . . . . . . . . . . . . . . . . . . . . 23

10 Dyson's renormalization formulas for Green's functions . . . . . . . . . . . . . . . . . . . . . . 30

Lecture V - Hopf algebra of Feynman graphs and combinatorial groups of renormalization 33

11 Connes-Kreimer Hopf algebra of Feynaman graphs and diffeographisms . . . . . . . . . . . . . . . . . . 33 
Aknowledgments. These lectures are based on a course for Ph.D. students in mathematics, held at Université Lyon 1 in spring 2006, by Alessandra Frabetti and Denis Perrot. Thanks Denis!

During the Summer School Geometric and Topological Methods for Quantum Field Theory, many students made interesting questions and comments which greatly helped the writing of these notes. Thanks to all of them!

\section{Lecture I - Groups and Hopf algebras}

In this lecture we review the classical duality between groups and Hopf algebras of certein types. Details can be found for instance in [17].

\section{Algebras of representative functions}

Let $G$ be a group, for instance a group of real or complex matrices, a topological or a Lie group. Let

$$
F(G)=\{f: G \longrightarrow \mathbb{C}(\text { or } \mathbb{R})\}
$$

denote the set of functions on $G$, eventually continuous or differentiable. Then $F(G)$ has a lot of algebraic structures, that we describe in details.

1.1 - Product. The natural vector space $F(G)$ is a unital associative and commutative algebra over $\mathbb{C}$, with product $(f g)(x)=f(x) g(x)$, where $f, g \in F(G)$ and $x \in G$, and unit given by the constant function $1(x)=1$.

1.2 - Coproduct. For any $f \in F(G)$, the group law $G \times G \stackrel{\cdot}{\longrightarrow} G$ induces an element $\Delta f \in F(G \times G)$ defined by $\Delta f(x, y)=f(x \cdot y)$. Can we characterise the algebra $F(G \times G)=\{f: G \times G \longrightarrow \mathbb{C}\}$ starting from $F(G)$ ?

Of course, we can consider the tensor product

$$
F(G) \otimes F(G)=\left\{\sum_{\text {finite }} f_{i} \otimes g_{i}, f_{i}, g_{i} \in F(G)\right\},
$$

with componentwise product $\left(f_{1} \otimes g_{1}\right)\left(f_{2} \otimes g_{2}\right)=f_{1} g_{1} \otimes f_{2} g_{2}$, but in general this algebra is a strict subalgebra of $F(G \times G)=\left\{\sum_{\text {infinite }} f_{i} \otimes g_{i}\right\}$ (it is equal for finite groups). For example, $f(x, y)=\exp (x+y) \in F(G) \otimes F(G)$, but $f(x, y)=\exp (x y) \notin F(G) \otimes F(G)$. Similarly, if $\delta(x, y)$ is the function equal to 1 when $x=y$ and equal to 0 when $x \neq y$, then $\delta \notin F(G) \otimes F(G)$. To avoid this problem we could use the completed or topological tensor product $\hat{\otimes}$ such that $F(G) \hat{\otimes} F(G)=F(G \times G)$. However this tensor product is difficult to handle, and for our purpuse we want to avoid it. In alternative, we can consider the subalgebras $R(G)$ of $F(G)$ such that $R(G) \otimes R(G)=R(G \times G)$. Such algebras are of course much easier to describe then a completed tensor product. For our purpuse, we are interested in the case when one of these subalgebras is big enough to describe completely the group. That is, it does not loose too much informations about the group with respect to $F(G)$. This condition will be specified later on.

Let us then suppose that there exists a subalgebra $R(G) \subset F(G)$ such that $R(G) \otimes R(G)=R(G \times G)$. Then, the group law $G \times G \stackrel{\cdot}{\longrightarrow} G$ induces a coproduct $\Delta: R(G) \longrightarrow R(G) \otimes R(G)$ defined by $\Delta f(x, y)=f(x \cdot y)$. We denote it by $\Delta f=\sum_{\text {finite }} f_{(1)} \otimes f_{(2)}$. The coproduct has two main properties:

1. $\Delta$ is a homomorphism of algebras, in fact

$$
\Delta(f g)(x, y)=(f g)(x \cdot y)=f(x \cdot y) g(x \cdot y)=\Delta f(x, y) \Delta g(x, y)
$$

that is $\Delta(f g)=\Delta(f) \Delta(g)$. This can also be expressed as $\sum(f g)_{1} \otimes(f g)_{2}=\sum f_{1} g_{1} \otimes f_{2} g_{2}$.

2. $\Delta$ is coassociative, that is $(\Delta \otimes \operatorname{Id}) \Delta=(\operatorname{Id} \otimes \Delta) \Delta$, because of the associativity $(x \cdot y) \cdot z=x \cdot(y \cdot z)$ of the group law in $G$. 
1.3 - Counit. The neutral element $e$ of the group $G$ induces a counit $\varepsilon: R(G) \longrightarrow \mathbb{C}$ defined by $\varepsilon(f)=f(e)$. The counit has two main properties:

1. $\varepsilon$ is a homomorphism of algebras, in fact

$$
\varepsilon(f g)=(f g)(e)=f(e) g(e)=\varepsilon(f) \varepsilon(g) .
$$

2. $\varepsilon$ satisfies the equality $\sum f_{(1)} \varepsilon\left(f_{(2)}\right)=\sum \varepsilon\left(f_{(1)}\right) f_{(2)}$, induced by the equality $x \cdot e=x=e \cdot x$ in $G$.

1.4 - Antipode. The operation of inversion in $G$, that is $x \rightarrow x^{-1}$, induces the antipode $S: R(G) \longrightarrow R(G)$ defined by $S(f)(x)=f\left(x^{-1}\right)$. The counit has four main properties:

1. $S$ is a homomorphism of algebras, in fact

$$
S(f g)(x)=(f g)\left(x^{-1}\right)=f\left(x^{-1}\right) g\left(x^{-1}\right)=S(f)(x) S(g)(x) .
$$

2. $S$ satisfies the 5-terms equality $m(S \otimes \operatorname{Id}) \Delta=u \varepsilon=m(\operatorname{Id} \otimes S) \Delta$, where $m: R(G) \otimes R(G) \longrightarrow R(G)$ denotes the product and $u: \mathbb{C} \longrightarrow R(G)$ denotes the unit. This is induced by the equality $x \cdot x^{-1}=e=x^{-1} \cdot x$ in $G$.

3. $S$ is anti-comultiplicative, that is $\Delta \circ S=(S \otimes S) \circ \tau \circ \Delta$, where $\tau(f \otimes g)=g \otimes f$ is the twist operator. This property is induced by the equality $(x \cdot y)^{-1}=y^{-1} \cdot x^{-1}$ in $G$.

4. $S$ is nilpotent, that is $S \circ S=\mathrm{Id}$, because of the identity $\left(x^{-1}\right)^{-1}=x$ in $G$.

1.5 - Abelian groups. Finally, $G$ is abelian, that is $x \cdot y=y \cdot x$ for all $x, y \in G$, if and only if the coproduct is cocommutative, that is $\Delta=\Delta \circ \tau$, i.e. $\sum f_{(1)} \otimes f_{(2)}=\sum f_{(2)} \otimes f_{(1)}$.

1.6 - Hopf algebras. A unital, associative and commutative algebra $\mathcal{H}$ endowed with a coproduct $\Delta$, a counit $\varepsilon$ and an antipode $S$, satisfying all the properties listed above, is called a commutative Hopf algebra.

In conclusion, we just showed that if $G$ is a (topological) group, and $R(G)$ is a subalgebra of (continuous) functions on $G$ such that $R(G) \otimes R(G)=R(G \times G)$, and sufficiently big to contain the image of $\Delta$ and of $S$, then $R(G)$ is a commutative Hopf algebra. Moreover, $R(G)$ is cocommutative if and only if $G$ is abelian.

1.7 - Representative functions. We now turn to the existence of such a Hopf algebra $R(G)$. If $G$ is a finite group, then the largest such algebra is simply the linear dual $R(G)=F(G)=(\mathbb{C} G)^{*}$ of the group algebra.

If $G$ is a topological group, then the condition $R(G) \otimes R(G)=R(G \times G)$ roughly forces $R(G)$ to be a polynomial algebra, or a quotient of it. The generators are the coordinate functions on the group, but we do not always know how to find them.

For compact Lie groups, $R(G)$ always exists, and we can be more precise. We say that a function $f: G \longrightarrow \mathbb{C}$ is representative if there exist a finite number of functions $f_{1}, \ldots, f_{k}$ such that any translation of $f$ is a linear combination of them. If we denote by $\left(L_{x} f\right)(y)=f(x \cdot y)$ the left translation of $f$ by $x \in G$, this means that $L_{x} f=\sum l_{i}(x) f_{i}$. Call $R(G)$ the set of all representative functions on $G$. Then, using representation theory, and in particular Peter-Weyl Theorem, one can show the following facts:

1. $R(G) \otimes R(G)=R(G \times G)$;

2. $R(G)$ is dense in the set of continuous functions;

3. as an algebra, $R(G)$ is generated by the matrix elements of all the representations of $G$ of finite dimension;

4. $R(G)$ is also generated by the matrix elements of one faithful representation of $G$, therefore it is finitely generated.

Moreover, for compact Lie groups, the algebra $R(G)$ has two additional structures:

1. because the group $G$ is a real manifold, and the functions have complex values, $R(G)$ has an involution, that is a map $*: R(G) \longrightarrow R(G)$ such that $\left(f^{*}\right)^{*}=f$ and $(f g)^{*}=g^{*} f^{*}$; 
2. because $G$ is compact, $R(G)$ has a Haar measure, that is, a linear map $\mu: R(G) \longrightarrow \mathbb{R}$ such that $\mu\left(a a^{*}\right)>0$ for all $a \neq 0$.

Similar results hold in general for groups of matrices, even if they are complex manifolds, and even if they are not compact. In particular, the algebra generated by the matrix elements of one faithful representation of $G$ satisfies the required properties.

For other groups then those of matrices, a suitable algebra $R(G)$ can exist, but there is no general procedure to find it. The best hint is to look for a faithful representation, eventually with infinite dimension. This may work also for groups which are not locally compact, as shown in the examples (2.8) and (2.9), but in general not for groups of diffeomorphisms on a manifold.

\section{Examples}

2.1 - Complex affine plane. Let $G=\left(\mathbb{C}^{n},+\right)$ be the additive group of the complex affine plane. A complex group is supposed to be a holomorphic manifold. The functions are also supposed to be holomorphic, that is they do not depend on the complex conjugate of the variables. The map

$$
\begin{aligned}
\rho:\left(\mathbb{C}^{n},+\right) & \longrightarrow G L_{n+1}(\mathbb{C})=\operatorname{Aut}\left(\mathbb{C}^{n+1}\right) \\
\left(t_{1}, \ldots, t_{n}\right) & \mapsto\left(\begin{array}{cccc}
1 & t_{1} & \ldots & t_{n} \\
0 & 1 & \ldots & 0 \\
& & \ldots & \\
0 & 0 & \ldots & 1
\end{array}\right)
\end{aligned}
$$

is a faithful representation, in fact

$$
\begin{aligned}
\rho\left(\left(t_{1}, \ldots, t_{n}\right)+\left(s_{1}, \ldots, s_{n}\right)\right) & =\left(\begin{array}{ccccc}
1 & t_{1}+s_{1} & \ldots & t_{n}+s_{n} \\
0 & 1 & \ldots & 0 \\
& & \ldots & \\
0 & 0 & \ldots & 1
\end{array}\right) \\
& =\left(\begin{array}{cccc}
1 & t_{1} & \ldots & t_{n} \\
0 & 1 & \ldots & 0 \\
& & \ldots & \\
0 & 0 & \ldots & 1
\end{array}\right)\left(\begin{array}{cccc}
1 & s_{1} & \ldots & s_{n} \\
0 & 1 & \ldots & 0 \\
& & \ldots & \\
0 & 0 & \ldots & 1
\end{array}\right)=\rho\left(t_{1}, \ldots, t_{n}\right) \rho\left(s_{1}, \ldots, s_{n}\right) .
\end{aligned}
$$

Therefore, there are $n$ local coordinates $x_{i}\left(t_{1}, \ldots, t_{n}\right)=t_{i}$, for $i=1, \ldots, n$, which are free of mutual relations. Hence the algebra of local coordinates on the affine line is the polynomial ring $R\left(\mathbb{C}^{n},+\right)=\mathbb{C}\left[x_{1}, \ldots, x_{n}\right]$. The Hopf structure is the following:

- Coproduct: $\Delta x_{i}=x_{i} \otimes 1+1 \otimes x_{i}$ and $\Delta 1=1 \otimes 1$. The group is abelian and the coproduct is indeed cocommutative.

- Counit: $\varepsilon\left(x_{i}\right)=x(0)=0$, and $\varepsilon(1)=1$.

- Antipode: $S x_{i}=-x_{i}$ and $S 1=1$.

This Hopf algebra is usually called the unshuffle Hopf algebra, because the coproduct on a generic monomial

$$
\Delta\left(x_{i_{1}} \cdots x_{i_{l}}\right)=\sum_{p+q=l} \sum_{\sigma \in \Sigma_{p, q}} x_{\sigma\left(i_{1}\right)} \cdots x_{\sigma\left(i_{p}\right)} \otimes x_{\sigma\left(i_{p+1}\right)} \cdots x_{\sigma\left(i_{p+q}\right)}
$$

makes use of the shuffle permutations $\sigma \in \Sigma_{p, q}$, that is the permutations of $\Sigma_{p+q}$ such that $\sigma\left(i_{1}\right)<\cdots<\sigma\left(i_{p}\right)$ and $\sigma\left(i_{p+1}\right)<\cdots<\sigma\left(i_{p+q}\right)$.

2.2 - Real affine plane. Let $G=\left(\mathbb{R}^{n},+\right)$ be the additive group of the real affine plane. A real group is supposed to be a differentiable manifold. The functions with values in $\mathbb{C}$ are the complexification of the functions with values in $\mathbb{R}$, that is, $R_{\mathbb{C}}(G)=R_{\mathbb{R}}(G) \otimes \mathbb{C}$. In principle, then, the functions depend also on the complex conjugates, but the generators must be real: we expect that the algebra $R_{\mathbb{C}}(G)$ has an involution $*$. In fact, we have the following results: 
- Real functions: the map

$$
\begin{aligned}
\rho:\left(\mathbb{R}^{n},+\right) & \longrightarrow G L_{n+1}(\mathbb{R})=\operatorname{Aut}\left(\mathbb{R}^{n+1}\right) \\
\left(t_{1}, \ldots, t_{n}\right) & \mapsto\left(\begin{array}{cccc}
1 & t_{1} & \ldots & t_{n} \\
0 & 1 & \ldots & 0 \\
& & \ldots & \\
0 & 0 & \ldots & 1
\end{array}\right)
\end{aligned}
$$

is a faithful representation. The local coordinates are $x_{i}\left(t_{1}, \ldots, t_{n}\right)=t_{i}$, for $i=1, \ldots, n$, and the algebra of real local coordinates is the polynomial ring $R_{\mathbb{R}}\left(\mathbb{R}^{n},+\right)=\mathbb{R}\left[x_{1}, \ldots, x_{n}\right]$. The Hopf structure is exactely as in the previous example.

- Complex functions: complex faithful representation as before, but local coordinates $x_{i}\left(t_{1}, \ldots, t_{n}\right)=t_{i}$ subject to an involution defined by $x_{i}^{*}\left(t_{1}, \ldots, t_{n}\right)=\overline{t_{i}}$ and such that $x_{i}^{*}=x_{i}$. Then the algebra of complex local coordinates is the quotient

$$
R_{\mathbb{C}}\left(\mathbb{R}^{n},+\right)=\frac{\mathbb{C}\left[x_{1}, x_{1}^{*}, \ldots, x_{n}, x_{n}^{*}\right]}{\left\langle x_{i}^{*}-x_{i}, i=1, \ldots, n\right\rangle},
$$

which is isomorphic to $\mathbb{C}\left[x_{1}, \ldots, x_{n}\right]$ as an algebra, but not as an algebra with involution. Of course the Hopf structure is always the same.

\section{3 - Complex simple linear group. The group}

$$
S L(2, \mathbb{C})=\left\{M=\left(\begin{array}{ll}
m_{11} & m_{12} \\
m_{21} & m_{22}
\end{array}\right) \in M_{2}(\mathbb{C}), \operatorname{det} M=m_{11} m_{22}-m_{12} m_{21}=1\right\}
$$

has a lot of finite-dimensional representations, and the smallest faithful one is the identity

$$
\begin{aligned}
\rho=\mathrm{Id}: & S L(2, \mathbb{C}) \longrightarrow G L_{2}(\mathbb{C}) \\
& M \mapsto\left(\begin{array}{ll}
m_{11}=a(M) & m_{12}=b(M) \\
m_{21}=c(M) & m_{22}=d(M)
\end{array}\right) .
\end{aligned}
$$

Therefore there are 4 local coordinates $a, b, c, d: S L(2, \mathbb{C}) \longrightarrow \mathbb{C}$, given by $a(M)=m_{11}$, etc, related by det $M=1$. Hence the algebra of local coordinates of $S L(2, \mathbb{C})$ is the quotient

$$
R(S L(2, \mathbb{C}))=\frac{\mathbb{C}[a, b, c, d]}{\langle a d-b c-1\rangle} .
$$

The Hopf structure is the following:

- Coproduct: $\Delta f(M, N)=f(M N)$, therefore

$$
\begin{array}{ll}
\Delta a=a \otimes a+b \otimes c & \Delta b=a \otimes b+b \otimes d \\
\Delta c=c \otimes a+d \otimes c & \Delta d=c \otimes b+d \otimes d
\end{array}
$$

To shorten the notation, we can write $\Delta\left(\begin{array}{ll}a & b \\ c & d\end{array}\right)=\left(\begin{array}{ll}a & b \\ c & d\end{array}\right) \otimes\left(\begin{array}{ll}a & b \\ c & d\end{array}\right)$.

- Counit: $\varepsilon(f)=f(1)$, hence $\varepsilon\left(\begin{array}{ll}a & b \\ c & d\end{array}\right)=\left(\begin{array}{ll}1 & 0 \\ 0 & 1\end{array}\right)$.

- Antipode: $S f(M)=f\left(M^{-1}\right)$, therefore $S\left(\begin{array}{ll}a & b \\ c & d\end{array}\right)=\left(\begin{array}{cc}d & -b \\ -c & a\end{array}\right)$.

2.4 - Complex general linear group. For the group

$$
G L(2, \mathbb{C})=\left\{M \in M_{2}(\mathbb{C}), \operatorname{det} M \neq 0\right\},
$$


the identity $G L(2, \mathbb{C}) \longrightarrow G L(2, \mathbb{C}) \equiv \operatorname{Aut}\left(\mathbb{C}^{2}\right)$ is of course a faithful representation. We have then 4 local coordinates as for $S L(2, \mathbb{C})$. However this time they satisfy the condition det $M \neq 0$ which is not closed. To express this relation we use a trick: since $\operatorname{det} M \neq 0$ if and only if there exists the inverse of det $M$, we add a variable $t(M)=(\operatorname{det} M)^{-1}$. Therefore the algebra of local coordinates of $G L(2, \mathbb{C})$ is the quotient

$$
R(G L(2, \mathbb{C}))=\frac{\mathbb{C}[a, b, c, d, t]}{\langle(a d-b c) t-1\rangle} .
$$

The Hopf structure is the same as that of $S L(2, \mathbb{C})$ on the local coordinates $a, b, c, d$, and on the new variable $t$ is given by

- Coproduct: since $\Delta t(M, N)=t(M N)=(\operatorname{det}(M N))^{-1}=(\operatorname{det} M)^{-1}(\operatorname{det} N)^{-1}=t(M) t(N)$, we have $\Delta t=t \otimes t$.

- Counit: $\varepsilon(t)=t(1)=1$.

- Antipode: $S t(M)=t\left(M^{-1}\right)=\left(\operatorname{det}\left(M^{-1}\right)\right)^{-1}=\operatorname{det} M$, therefore $S t=a d-b c$.

2.5 - Simple unitary group. The group

$$
S U(2)=\left\{M \in M_{2}(\mathbb{C}), \operatorname{det} M=1, M^{-1}=\bar{M}^{t}\right\}
$$

is a real group, infact it is one real form of $S L(2, \mathbb{C})$, the other one being $S L(2, \mathbb{R})$, and it is also the maximal compact subgroup of $S L(2, \mathbb{C})$. As a real manifold, $S U(2)$ is isomorphic to the 3 -dimensional sphere $S^{3}$, in fact

$$
M=\left(\begin{array}{ll}
a & b \\
c & d
\end{array}\right) \in S U(2) \quad \Longleftrightarrow \quad \begin{gathered}
a d-b c=1 \\
\bar{a}=d, \bar{b}=c
\end{gathered} \quad \Longleftrightarrow \quad M=\left(\begin{array}{cc}
a & b \\
-\bar{b} & \bar{a}
\end{array}\right) \quad \text { with } a \bar{a}+b \bar{b}=1 .
$$

If we set $a=x+i y$ and $b=u+i v$, with $x, y, u, v \in \mathbb{R}$, we then have

$$
a \bar{a}+b \bar{b}=1 \quad \Longleftrightarrow \quad x^{2}+y^{2}+u^{2}+v^{2}=1 \quad \text { in } \mathbb{R}^{4} \quad \Longleftrightarrow \quad(x, y, u, v) \in S^{3} .
$$

We then expect that the algebra of complex functions on $S U(2)$ has an involution:

$$
R(S U(2))=\frac{\mathbb{C}\left[a, b, c, d, a^{*}, b^{*}, c^{*}, d^{*}\right]}{\left\langle a^{*}-d, b^{*}+c, a d-b c-1\right\rangle} \cong \frac{\mathbb{C}\left[a, b, a^{*}, b^{*}\right]}{\left\langle a a^{*}+b b^{*}-1\right\rangle} .
$$

The Hopf structure is the same as that of $S L(2, \mathbb{C})$, but expressed in terms of the proper coordinate functions of $S U(2)$, that is:

- Coproduct: $\Delta\left(\begin{array}{cc}a & b \\ -b^{*} & a^{*}\end{array}\right)=\left(\begin{array}{cc}a & b \\ -b^{*} & a^{*}\end{array}\right) \otimes\left(\begin{array}{cc}a & b \\ -b^{*} & a^{*}\end{array}\right)$.

- Counit: $\varepsilon\left(\begin{array}{cc}a & b \\ -b^{*} & a^{*}\end{array}\right)=\left(\begin{array}{ll}1 & 0 \\ 0 & 1\end{array}\right)$.

- Antipode: $S\left(\begin{array}{cc}a & b \\ -b^{*} & a^{*}\end{array}\right)=\left(\begin{array}{cc}a^{*} & -b \\ b^{*} & a\end{array}\right)$.

2.6 - Exercise: Heisenberg group. The Heisenberg group $H_{3}$ is the group of complex $3 \times 3$ (upper) triangular matrices with all the diagonal elements equal to 1 , that is

$$
H_{3}=\left\{\left(\begin{array}{lll}
1 & a & b \\
0 & 1 & c \\
0 & 0 & 1
\end{array}\right) \in G L(3, \mathbb{C})\right\} .
$$

Describe the Hopf algebra of complex representative (algebraic) functions on $\mathrm{H}_{3}$. 
2.7 - Exercise: Euclidean group. The group of rotations on the plane $\mathbb{R}^{2}$ is the special orthogonal group

$$
S O(2, \mathbb{R})=\left\{A \in G L(2, \mathbb{R}), \operatorname{det} A=1, A^{-1}=A^{t}\right\} .
$$

The group of rotations acts on the group of translations $T_{2}=\left(\mathbb{R}^{2},+\right)$ as a product $A v$ of a matrix $A \in$ $S O(2, \mathbb{R})$ by a vector $v \in \mathbb{R}^{2}$.

The Euclidean group is the semi-direct product $E_{2}=T_{2} \rtimes S O(2, \mathbb{R})$. That is, $E_{2}$ is the set of all the couples $(v, A) \in T_{2} \times S O(2, \mathbb{R})$, with the group law

$$
(v, A) \cdot(u, B):=(v+A u, A B) .
$$

1. Describe the Hopf algebra of real representative functions on $S O(2, \mathbb{R})$.

2. Find a real faithful representation of $T_{2}$ of dimension 3 .

3. Describe the Hopf algebra of real representative functions on $E_{2}$.

2.8 - Group of invertible formal series. The set

$$
G^{\mathrm{inv}}(\mathbb{C})=\left\{f(z)=\sum_{n=0}^{\infty} f_{n} z^{n}, f_{n} \in \mathbb{C}, f_{0}=1\right\}
$$

of formal series in one variable, with constant term equal to 1, is an Abelian group with

- product: $(f g)(z)=f(z) g(z)=\sum_{n=0}^{\infty}\left(\sum_{p+q=n} f_{p} g_{q}\right) z^{n}$

- unit: $1(z)=1$;

- inverse: by recursion, in fact $\left(f f^{-1}\right)(z)=\sum_{n=0}^{\infty}\left(\sum_{p+q=n} f_{p}\left(f^{-1}\right)_{q}\right) z^{n}=1$ if and only if

$$
\begin{array}{ll}
\text { for } n=0 & f_{0}\left(f^{-1}\right)_{0}=1 \quad \Leftrightarrow \quad\left(f^{-1}\right)_{0}=1 \quad \Leftrightarrow \quad f^{-1} \in G^{\text {inv }}(\mathbb{C}), \\
\text { for } n \geq 1 & \sum_{p=0}^{n} f_{p}\left(f^{-1}\right)_{n-p}=f_{0}\left(f^{-1}\right)_{n}+f_{1}\left(f^{-1}\right)_{n-p}+\cdots+f_{n-1}\left(f^{-1}\right)_{1}+f_{n}\left(f^{-1}\right)_{0}=0 \\
& \text { that is }\left(f^{-1}\right)_{1}=-f_{1},\left(f^{-1}\right)_{2}=f_{1}^{2}-f_{2}, \ldots
\end{array}
$$

This group has many finite-dimensional representations, of the form

$$
\begin{gathered}
\rho: G^{\text {inv }}(\mathbb{C}) \longrightarrow G L_{n}(\mathbb{C}) \\
f(z)=\sum_{n=0}^{\infty} f_{n} z^{n} \mapsto\left(\begin{array}{cccccc}
1 & f_{1} & f_{2} & f_{3} & \ldots & f_{n-1} \\
0 & 1 & f_{1} & f_{2} & \ldots & f_{n-2} \\
0 & 0 & 1 & f_{1} & \ldots & f_{n-3} \\
& & & \ldots & & \\
0 & 0 & & \ldots & & 1
\end{array}\right)
\end{gathered}
$$

but they are never faithful! To have a faithful representation, we need to consider the map

$$
\begin{array}{r}
\rho: G^{\mathrm{inv}}(\mathbb{C}) \longrightarrow G L_{\infty}(\mathbb{C})=\underset{\leftarrow}{\lim } G L_{n}(\mathbb{C}) \\
f(z) \mapsto\left(\begin{array}{ccccc}
1 & f_{1} & f_{2} & f_{3} & \ldots \\
0 & 1 & f_{1} & f_{2} & \ldots \\
0 & 0 & 1 & f_{1} & \ldots \\
& & \ldots & & \\
0 & 0 & \ldots & &
\end{array}\right)
\end{array}
$$


where $\lim G L_{n}(\mathbb{C})$ is the projective limit of the groups $\left(G L_{n}(\mathbb{C})\right)_{n}$, that is, the limit of the groups such that each $G \overleftarrow{L_{n}}(\mathbb{C})$ is identified with the quotient of $G L_{n+1}(\mathbb{C})$ by its last column and row.

Therefore there are infinitely many local coordinates $x_{n}: G^{\text {inv }}(\mathbb{C}) \longrightarrow \mathbb{C}$, given by $x_{n}(f)=f_{n}$, which are free one from each other. Hence the algebra of local coordinates of $G^{\text {inv }}(\mathbb{C})$ is the polynomial ring

$$
R\left(G^{\text {inv }}(\mathbb{C})\right)=\mathbb{C}\left[x_{1}, x_{2}, \ldots, x_{n}, \ldots\right] .
$$

The Hopf structure is the following (with $x_{0}=1$ ):

- Coproduct: $\Delta x_{n}=\sum_{k=0}^{n} x_{k} \otimes x_{n-k}$.

- Counit: $\varepsilon\left(x_{n}\right)=\delta(n, 0)$.

- Antipode: recursively, from the 5-terms identity. In fact, for any $n>0$ we have

$$
\varepsilon\left(x_{n}\right) 1=0=\sum_{k=0}^{n} S\left(x_{k}\right) x_{n-k}=S(1) x_{n}+S\left(x_{1}\right) x_{n-1}+S\left(x_{2}\right) x_{n-2}+\cdots+S\left(x_{n}\right) 1
$$

and since $S(1)=1$ we obtain $S\left(x_{n}\right)=-x_{n}-\sum_{k=1}^{n-1} S\left(x_{k}\right) x_{n-k}$.

This Hopf algebra is isomorphic to the so-called algebra of symmetric functions, cf. [20].

2.9 - Group of formal diffeomorphisms. The set

$$
G^{\mathrm{dif}}(\mathbb{C})=\left\{f(z)=\sum_{n=0}^{\infty} f_{n} z^{n+1}, f_{n} \in \mathbb{C}, f_{0}=1\right\}
$$

of formal series in one variable, with zero constant term and linear term equal to 1, is a (non-Abelian) group with

- product: given by the composition (or substitution)

$$
\begin{aligned}
(f \circ g)(z) & =f(g(z))=\sum_{n=0}^{\infty} f_{n} g(z)^{n} \\
& =z+\left(f_{1}+g_{1}\right) z^{2}+\left(f_{2}+2 f_{1} g_{1}+g_{2}\right) z^{3}+\left(f_{3}+3 f_{2} g_{1}+2 f_{1} g_{2}+f_{1} g_{1}^{2}+g_{3}\right) z^{4}+\mathcal{O}\left(z^{5}\right) .
\end{aligned}
$$

- unit: $\operatorname{id}(z)=z$;

- inverse: given by the by the reciprocal series $f^{-1}$, such that $f \circ f^{-1}=\mathrm{id}=f^{-1} \circ f$, which can be found recursively, using for instance Lagrange Formula, cf. [23].

This group also has many finite-dimensional representations, which are not faithful, and a faithful representation of infinite dimension:

$$
\begin{aligned}
& \rho: G^{\operatorname{dif}}(\mathbb{C}) \longrightarrow G L_{\infty}(\mathbb{C})=\lim _{\leftarrow} G L_{n}(\mathbb{C}) \\
& f(z) \mapsto\left(\begin{array}{ccccccc}
1 & f_{1} & f_{2} & f_{3} & f_{4} & \ldots \\
0 & 1 & 2 f_{1} & 2 f_{2}+f_{1}^{2} & 2 f_{3}+2 f_{1} f_{2} & \ldots \\
0 & 0 & 1 & 3 f_{1} & 3 f_{2}+3 f_{1}^{2} & \ldots \\
0 & 0 & 0 & 1 & 4 f_{1} & \cdots \\
0 & 0 & & \ldots & &
\end{array}\right) .
\end{aligned}
$$

Therefore there are infinitely many local coordinates $x_{n}: G^{\text {dif }}(\mathbb{C}) \longrightarrow \mathbb{C}$, given by $x_{n}(f)=f_{n}$, which are free one from each other. As in the previous example, the algebra of local coordinates of $G^{\mathrm{dif}}(\mathbb{C})$ is then the polynomial ring

$$
R\left(G^{\mathrm{dif}}(\mathbb{C})\right)=\mathbb{C}\left[x_{1}, x_{2}, \ldots\right] .
$$

The Hopf structure is the following (with $x_{0}=1$ ): 
- Coproduct: $\Delta x_{n}(f, g)=x_{n}(f \circ g)$, hence $\Delta x_{n}=x_{n} \otimes 1+1 \otimes x_{n}+\sum_{m=1}^{n-1} x_{m} \otimes \sum_{p_{0}+p_{1}+\cdots+p_{m}=n-m} x_{p_{0}, \ldots, p_{m} \geq 0} x_{p_{1}} \cdots x_{p_{m}}$.

- Counit: $\varepsilon\left(x_{n}\right)=\delta(n, 0)$.

- Antipode: recursively, using $S\left(x_{n}\right)=-x_{n}-\sum_{m=1}^{n-1} S\left(x_{m}\right) \sum_{\substack{p_{0}+p_{1}+\cdots+p_{m}=n-m \\ p_{0}, \ldots, p_{m} \geq 0}} x_{p_{0}} x_{p_{1}} \cdots x_{p_{m}}$.

This Hopf algebra is the so-called Fà̀ di Bruno Hopf algebra, because the computations of the coefficients of the Taylor expansion of the composition of two functions was firstly done by F. Faà di Bruno in [13] (in 1855!).

\section{Groups of characters and duality}

Let $\mathcal{H}$ be a commutative Hopf algebra over $\mathbb{C}$, with product $m$, unit $u$, coproduct $\Delta$, counit $\varepsilon$, antipode $S$ and eventually an involution $*$.

3.1 - Group of characters. We call character of the Hopf algebra $\mathcal{H}$ a linear map $\alpha: \mathcal{H} \longrightarrow \mathbb{C}$ such that

1. $\alpha$ is a homomorphism of algebras, i.e. $\alpha(a b)=\alpha(a) \alpha(b)$;

2. $\alpha$ is unital, i.e. $\alpha(1)=1$.

Call $G_{\mathcal{H}}=\operatorname{Hom}_{A l g}(\mathcal{H}, \mathbb{C})$ the set of characters of $\mathcal{H}$. Given two characters $\alpha, \beta \in G_{\mathcal{H}}$, we call convolution of $\alpha$ and $\beta$ the linear map $\alpha \star \beta: \mathcal{H} \longrightarrow \mathbb{C}$ defined by $\alpha \star \beta=m_{\mathbb{C}} \circ(\alpha \otimes \beta) \circ \Delta$, that is, $\alpha \star \beta(a)=\sum \alpha\left(a_{(1)}\right) \beta\left(a_{(2)}\right)$ for any $a \in \mathcal{H}$. Applying the definitions, it is easy to prove the following properties:

1. For any $\alpha, \beta \in G_{\mathcal{H}}$, the convolution $\alpha \star \beta$ is a unital algebra homomorphism, that is $\alpha \star \beta \in G_{\mathcal{H}}$.

2. The convolution product $G_{\mathcal{H}} \otimes G_{\mathcal{H}} \longrightarrow G_{\mathcal{H}}$ is associative.

3. The counit $\varepsilon: \mathcal{H} \longrightarrow \mathbb{C}$ is the unit of the convolution.

4. For any $\alpha \in G_{\mathcal{H}}$, the homomorphism $\alpha^{-1}=\alpha \circ S$ is the inverse of $\alpha$.

5. The convolution product is commutative if and only if the coproduct is cocommutative.

In other words, the set of characters $G_{\mathcal{H}}$ forms a group with the convolution product.

3.2 - Real subgroups. If $\mathcal{H}$ is a commutative Hopf algebra endowed with an involution $*: \mathcal{H} \longrightarrow \mathcal{H}$ compatible with the Hopf structure, in the sense that

$$
\begin{aligned}
(a b)^{*}=b^{*} a^{*}, & 1^{*}=1 \\
\Delta\left(a^{*}\right)=(\Delta a)^{*}, & \varepsilon\left(a^{*}\right)=\varepsilon(a), \quad S\left(a^{*}\right)=(S a)^{*},
\end{aligned}
$$

then the subset

$$
G_{\mathcal{H}}^{*}=\operatorname{Hom}_{* A l g}(\mathcal{H}, \mathbb{C})=\left\{\alpha \in G_{\mathcal{H}}, \alpha\left(a^{*}\right)=\overline{\alpha(a)}\right\}
$$

is a (real) subgroup of $G_{\mathcal{H}}$.

3.3 - Compact subgroups. If, furthermore, $\mathcal{H}$ is a commutative *Hopf algebra, finitely generated and endowed with a Haar measure compatible with the Hopf structure, that is, a linear map $\mu: \mathcal{H} \longrightarrow \mathbb{R}$ such that

$$
\begin{aligned}
& (\mu \otimes \operatorname{Id}) \Delta=(\operatorname{Id} \otimes \mu) \Delta=u \circ \mu, \\
& \mu\left(a a^{*}\right)>0 \quad \text { for all } a \neq 0,
\end{aligned}
$$

then $G_{\mathcal{H}}^{*}$ is a compact Lie group. 
3.4 - Comparision of $S L(2, \mathbb{C}), S L(2, \mathbb{R})$ and $S U(2)$. Consider the commutative algebra $\mathcal{H}=\frac{\mathbb{C}[a, b, c, d]}{\langle a d-b c-1\rangle}$.

If on $\mathcal{H}$ we consider the Hopf structure

$$
\begin{aligned}
\Delta\left(\begin{array}{ll}
a & b \\
c & d
\end{array}\right) & =\left(\begin{array}{ll}
a & b \\
c & d
\end{array}\right) \otimes\left(\begin{array}{ll}
a & b \\
c & d
\end{array}\right) \\
\varepsilon\left(\begin{array}{ll}
a & b \\
c & d
\end{array}\right) & =\left(\begin{array}{ll}
1 & 0 \\
0 & 1
\end{array}\right) \\
S\left(\begin{array}{ll}
a & b \\
c & d
\end{array}\right) & =\left(\begin{array}{cc}
d & -b \\
-c & a
\end{array}\right),
\end{aligned}
$$

then $G_{\mathcal{H}}=S L(2, \mathbb{C})$. If in addition we consider the involution

$$
\left(\begin{array}{ll}
a & b \\
c & d
\end{array}\right)^{*}=\left(\begin{array}{ll}
a & b \\
c & d
\end{array}\right)
$$

then $G_{\mathcal{H}}^{*}=S L(2, \mathbb{R})$. If, instead, we consider the involution

$$
\left(\begin{array}{ll}
a & b \\
c & d
\end{array}\right)^{*}=\left(\begin{array}{cc}
d & -c \\
-b & a
\end{array}\right)
$$

then $G_{\mathcal{H}}^{*}=S U(2)$.

3.5 - Duality. We have seen first how to associate a Hopf algebra to a group, through a functor $R$, and then how to associate a group to a Hopf algebra, through a functor $G$. In general, these two functors are adjoint one to each other, that is

$$
\operatorname{Hom}_{\text {Groups }}\left(G, G_{\mathcal{H}}\right) \cong \operatorname{Hom}_{\text {Alg }}(\mathcal{H}, R(G))
$$

Sometimes, these two functors are dual one to each other. In particular, we have the following results:

- Given a complex group $G$, and its Hopf algebra $R(G)$ of representative functions, the map

$$
\begin{aligned}
\Phi: G & \longrightarrow G_{R(G)}=\operatorname{Hom}_{A l g}(R(G), \mathbb{C}) \\
x & \mapsto \Phi_{x}: \mathbb{R}(G) \rightarrow \mathbb{C}, \Phi_{x}(f)=f(x)
\end{aligned}
$$

defines an isomorphism of groups to the characters group of $R(G)$. This result must be refined to the group $G_{R(G)}^{*}$ if $G$ is real. It is known as Tannaka duality for compact Lie groups.

- Viceversa, given a commutative Hopf algebra over $\mathbb{C}$, the complex group $G$ can be defined as the group of characters of $\mathcal{H}$, that is, by stating that its coordinate functions are given by $\mathcal{H}$. If the Hopf algebra $\mathcal{H}$ has an involution and a Haar measure, and it is finitely generated, then the map

$$
\begin{aligned}
\Psi: \mathcal{H} & \longrightarrow R\left(G^{*}(\mathcal{H})\right) \\
a & \mapsto \Psi_{a}: \operatorname{Hom}_{* A l g}(\mathcal{H}, \mathbb{C}) \rightarrow \mathbb{C}, \Psi_{a}(\alpha)=\alpha(a)
\end{aligned}
$$

defines an isomorphism of Hopf algebras. The underlying group is compact, and this result is known as the Krein duality.

3.6 - Algebraic and proalgebraic groups. As we saw in the most of the examples, the group structure of many groups does not depend on the field where the coefficients take value. This is the case of matrix groups, but also of the groups of formal series. Apart from the coefficients, such groups have in common the form of their coordinate ring, that is the Hopf algebra $\mathcal{H}$. They are better described as follows.

Given a commutative Hopf algebra $\mathcal{H}$ which is finitely generated, we call algebraic group associated to $\mathcal{H}$ the functor

$$
\begin{aligned}
G_{\mathcal{H}}: & \{\text { Commutative, associative algebras }\} \longrightarrow\{\text { Groups }\} \\
& A \mapsto G_{\mathcal{H}}(A)=\operatorname{Hom}_{A l g}(\mathcal{H}, A),
\end{aligned}
$$


where $G_{\mathcal{H}}(A)$ is a group with the convolution product. If $\mathcal{H}$ is not finitely generated, we call proalgebraic group the same functor.

In particular, all the matrix groups $S L_{n}, G L_{n}$, etc., can have matrix coefficients in any commutative algebra $A$, not only $\mathbb{C}$ or $\mathbb{R}$, and therefore are algebraic groups. Similarly, the groups of formal series $G^{\text {inv }}, G^{\text {dif }}$, with coefficients in any commutative algebra $A$, are proalgebraic groups.

\section{Lecture II - Review on field theory}

\section{Review of classical field theory}

In this section we briefly review the standard Lagrangian tools applied to fields, and the main examples of solutions of the Euler-Lagrange equations.

4.1 - Space-time. The space-time coordinates are points in the Minkowski space $\mathbb{R}^{1,3}$, that is, the space endowed with the flat diagonal metric $g=(1,-1,-1,-1)$. A transformation, called Wick's rotation, allows to reformulate the problems on the Euclidean space $\mathbb{R}^{4}$. For more generality, we then consider an Eucledian space $\mathbb{R}^{D}$ of dimension $D$, and we denote the space-time coordinates by $x=\left(x^{\mu}\right)$, with $\mu=0,1, \ldots, D-1$.

4.2 - Classical fields. A field is a section of a bundle on a base space. If the base space is flat, as in the case we consider here, a field is just a vector-valued function. By classical field, we mean a real function $\phi: \mathbb{R}^{D} \longrightarrow \mathbb{R}$ of class $C^{\infty}$, with compact support and rapidly decreasing. To be precise, we can take the function $\phi$ in the Schwartz space $\mathcal{S}\left(\mathbb{R}^{D}\right)$, that is, $\phi$ is a $C^{\infty}$ function such that all its derivatives $\partial_{\mu}^{n} \phi$ converge rapidly to zero for $|x| \rightarrow \infty$.

The observables of the system described by a field $\phi$, that is, the observable quantities, are real functionals $F$ of the field $\phi$, and what can be measured of these observables are the values $F(\phi) \in \mathbb{R}$. To determine all the observables it is enough to know the field itself.

When the field $\phi: \mathbb{R}^{D} \longrightarrow \mathbb{C}$ has complex (unreal) values, or vector values $\mathbb{C}^{4}$, it is called a wave function. In this case, what can be measured is not the value $\phi(x)$ itself, for any $x \in \mathbb{R}^{D}$, but rather the real value $|\phi(x)|^{2}$ which describes the probability to find the particle in the position $x$.

4.3 - Euler-Lagrange equation. A classical field is determined as the solution of a partial differential equation, called the field equation, which encodes its evolution. To any system is associated a Lagrangian density, that is a real function $\mathcal{L}: \mathbb{R}^{D} \longrightarrow \mathbb{R}, x \mapsto \mathcal{L}(x, \phi(x), \partial \phi(x))$, where $\partial \phi$ denotes the gradient of $\phi$. By Noether's theorem, the dynamics of the field $\phi$ is such that the symmetries of the field (i.e. the transformations which leave the Lagrangian invariant) are conserved. This conservation conditions are turned into a field equation by means of the action of the field $\phi$ : it is the functional $S$ of $\phi$ given by

$$
\phi \mapsto S[\phi]=\int_{\mathbb{R}^{D}} d^{D} x \mathcal{L}(x, \phi(x), \partial \phi(x)) .
$$

The action $S$ is stationary in $\phi \in \mathcal{S}\left(\mathbb{R}^{D}\right)$ if for any other function $\delta \phi \in \mathcal{S}\left(\mathbb{R}^{D}\right)$ we have $\frac{d}{d t} S[\phi+t \delta \phi]_{\mid t=0}=0$. Then, Hamilton's principle of least (or stationary) action states that a field $\phi$ satisfies the field equation if and only if the action $S$ is stationary in $\phi$. In terms of the Lagrangian, the field equation results into the so-called Euler-Lagrange equation

$$
\left[\frac{\partial \mathcal{L}}{\partial \phi}-\sum_{\mu} \partial_{\mu}\left(\frac{\partial \mathcal{L}}{\partial\left(\partial_{\mu} \phi\right)}\right)\right](x, \phi(x))=0 .
$$

This is the equation that we have to solve to find the classical field $\phi$. In general, it is a non-homogenous and non-linear partial differential equation, where the non-homogeneous terms appear if the system is not isolated, and the non-linear terms appear if the field is self-interacting.

For example, a field with Lagrangian density

$$
\mathcal{L}(x, \phi(x), \partial \phi(x))=\frac{1}{2}\left(\left|\partial_{\mu} \phi(x)\right|^{2}+m^{2} \phi(x)^{2}\right)-J(x) \phi(x)-\frac{\lambda}{3 !} \phi(x)^{3}-\frac{\mu}{4 !} \phi(x)^{4}
$$


is subject to the Euler-Lagrange equation

$$
\left(-\Delta+m^{2}\right) \phi(x)=J(x)+\frac{\lambda}{2} \phi(x)^{2}+\frac{\mu}{3 !} \phi(x)^{3},
$$

where we denote $\Delta \phi(x)=\sum_{\mu} \partial_{\mu}\left(\partial_{\mu} \phi(x)\right)$. This equation is called the Klein-Gordon equation, because the operator $-\Delta+m^{2}$ is called the Klein-Gordon operator.

4.4 - Free and interacting Lagrangian. A generic relativistic particle with mass $m$, described by a field $\phi$, can have a Lagrangian density of the form

$$
\mathcal{L}(x, \phi(x), \partial \phi(x))=\frac{1}{2} \phi^{t}(x) A \phi(x)-J(x) \phi(x)-\frac{\lambda}{3 !} \phi(x)^{3}-\frac{\mu}{4 !} \phi(x)^{4},
$$

where $A$ is a differential operator such as the Dirac operator or the Laplacian, typically summed up with the operator of multiplication by the mass or its square. The term $\frac{1}{2} \phi^{t} A \phi$ (quadratic in $\phi$ ) is the kinetic term. It is also called free Lagrangian density, and denoted by $\mathcal{L}_{0}$.

The field $J$ is an external field, which may represent a source for the field $\phi$. If $J=0$, the system described by $\phi$ is isolated, that is, it is placed in the vacuum. The term of the Lagrangian containing $J$ (linear in $\phi$ ) is the same for and field theory.

The parameters $\lambda, \mu$ are called coupling constants, because they express the self-interactions of the field. They are usually measurable parameters such as the electric charge or the flavour, but can also be unphysical parameters added for convenience. The sum of the terms which are non-quadratic in $\phi$ (and non-linear) is called interacting Lagrangian density, and denoted by $\mathcal{L}_{\text {int }}$.

4.5 - Free fields. A free field, that we shall denote by $\phi_{0}$, has the dynamics of a free Lagrangian $\mathcal{L}\left(\phi_{0}\right)=$ $\frac{1}{2} \phi_{0}^{t} A \phi_{0}-J \phi_{0}$. The Euler-Lagrange equation is easily written in the form

$$
A \phi_{0}(x)=J(x) .
$$

The general solution of this equation is well known to be the sum $\phi_{0}^{g}+\phi_{0}^{p}$ of the general solution of the homogeneous equation $A \phi_{0}^{g}(x)=0$, and a particular solution $\phi_{0}^{p}(x)$ of the non-homogeneous one. In the Minkowski space-time the function $\phi_{0}^{g}$ is a wave (superposition of plane waves), in the Euclidean space-time the formal solution $\phi_{0}^{g}$ is not a Schwartz function and we do not consider it. Therefore the function $\phi_{0}$ is the convolution

$$
\phi_{0}(x)=\int d^{D} y G_{0}(x-y) J(y)
$$

where $G_{0}(x)$ is the Green's function of the operator $A$, that is, the distribution such that $A G_{0}(x)=\delta(x)$. The physical interpretation of the convolution is that from each point $y$ of its support, the source $J$ affects the field $\phi$ at the position $x$ through the action of $G_{0}(x-y)$, which is then regarded as the field propagator.

For instance, if $A=-\Delta+m^{2}$ is the Klein-Gordon operator, the Green's function $G_{0}$ is the distribution defined by the Fourier transformation

$$
G_{0}(x-y)=\int_{\mathbb{R}^{D}} \frac{d^{D} p}{(2 \pi)^{D}} \frac{1}{p^{2}+m^{2}} e^{-i p \cdot(x-y} .
$$

4.6 - Self-interacting fields. A field $\phi$ with Lagrangian density of the form (4.4.1) satisfies the EulerLagrange equation

$$
A \phi(x)=J(x)+\frac{\lambda}{2} \phi(x)^{2}+\frac{\mu}{3 !} \phi(x)^{3} .
$$

This differential equation is non-linear, and in general can not be solved exactely. If the coupling constants $\lambda$ and $\mu$ are suitably small, we solve it perturbatively, that is, we regard the interacting terms as perturbations of the free ones. In fact, the Euler-Lagrange equation can be expressed as a recursive equation

$$
\phi(x)=\int_{\mathbb{R}^{D}} d^{D} y G_{0}(x-y)\left[J(y)+\frac{\lambda}{2} \phi(y)^{2}+\frac{\mu}{3 !} \phi(y)^{3}\right],
$$


where $G_{0}$ is the Green's function of $A$. This equation can then be solved as a formal series in the powers of $\lambda$ and $\mu$.

For instance, let us consider the simpliest Lagrangian (4.4.1) with $\mu=0$, whose Euler-Lagrange equation is

$$
\phi(x)=\int_{\mathbb{R}^{D}} d^{D} y G_{0}(x-y)\left[J(y)+\frac{\lambda}{2} \phi(y)^{2}\right] .
$$

If on the right hand-side of Eq. (4.6.1) we replace $\phi(y)$ by its value, and we repeat the substitutions recursively, we obtain the following perturbative solution:

$$
\begin{aligned}
\phi(x) & =\int d^{D} y G_{0}(x-y) J(y) \\
+ & \frac{\lambda}{2} \int d^{D} y d^{D} z d^{D} u G_{0}(x-y) G_{0}(y-z) G_{0}(y-u) J(z) J(u) \\
+ & \frac{2 \lambda^{2}}{4} \int d^{D} y d^{D} z d^{D} u d^{D} v d^{D} w G_{0}(x-y) G_{0}(y-z) G_{0}(y-u) G_{0}(z-v) G_{0}(z-w) \\
& \times J(z) J(u) J(v) J(w)
\end{aligned}
$$

which describes the self-interacting field in presence of an external field $J$.

4.7 - Conclusion. To summerize, a typical classical field $\phi$ with Lagrangian density of the form

$$
\mathcal{L}(\phi)=\frac{1}{2} \phi^{t} A \phi-J(x) \phi(x)-\frac{\lambda}{3 !} \phi(x)^{3},
$$

can be described perturbatively as a formal series

$$
\phi(x)=\sum_{n=0}^{\infty} \lambda^{n} \phi_{n}(x)
$$

in the powers of the coupling constant $\lambda$. Each coefficient $\phi_{n}(x)$ is a finite sum of integrals involving only the field propagator and the source. We describe these coefficients in Lecture III, using Feynman graphs.

\section{Review of quantum field theory}

In this section we briefly review the standard tools to describe quantum fields.

5.1 - Minkowski versus Euclidean approach. In the Minkowski space-time coordinates, the quantization procedure is the so-called canonical quantization, based on the principle that the observables of a quantum system are self-adjoint operators acting on a Hilbert space whose elements are the states in which the system can be found. The probability that the measurerement of an observable $F$ is the value carried by a state $v$ is given by the expectation value $\langle v|F| v\rangle \in \mathbb{R}$. In this procedure, the quantum fields are field operators, which must be defined together with the Hilbert space of states on which they act.

A standard way to deal with quantum fields is to Wick rotate the time, through the transformation $t \mapsto-i t$, and therefore transform the Minkowski space-time into a Euclidean space. The quantum fields are then treated as statistical fields, that is, classical fields or wave functions $\phi$ which fluctuate around their expectation values. The result is equivalent to that of the Minkowski approach, and this quantization procedure is the so-called path integral quantization.

5.2 - Green's functions through path integrals. The first interesting expectation value is the mean value $\langle\phi(x)\rangle$ of the field $\phi$ at the point $x$. More generally, we wish to compute the Green's functions $\left\langle\phi\left(x_{1}\right) \cdots \phi\left(x_{k}\right)\right\rangle$, which represent the probability that the quantum field $\phi$ moves from the point $x_{k}$ to $x_{k-1}$ and so on, and reaches $x_{1}$. 
A quantum field does not properly satisfy the principle of stationary action, but can be interpretated as a fluctuation around the classical solution of the Euler-Lagrange equation. On the Euclidean space, the probability to observe the quantum field at the value $\phi$ is proportional to $\exp \left(-\frac{S[\phi]}{\hbar}\right){ }^{1}$, where $\hbar=\frac{h}{2 \pi}$ is the reduced Planck's constant. When $\hbar \rightarrow 0$ (classical limit), we recover a maximal probability to find the field $\phi$ at the minimum of the action, that is, to recover the classical solution of Euler-Lagrange equation. The Green's functions can then be computed as the path integrals

$$
\left\langle\phi\left(x_{1}\right) \cdots \phi\left(x_{k}\right)\right\rangle=\frac{\left.\int d \phi \phi\left(x_{1}\right) \cdots \phi\left(x_{k}\right) e^{-\frac{S[\phi]}{\hbar}}\right|_{J=0}}{\left.\int d \phi e^{-\frac{S[\phi]}{\hbar}}\right|_{J=0}} .
$$

This approach presents a major problem: on the infinite dimensional set of classical fields, that we may fix as the Schwartz space $\mathcal{S}\left(\mathbb{R}^{D}\right)$, for $D>1$ there is no measure $d \phi$ suitable to give a meaning to such an integral. (For $D=1$ the problem is solved on continuous functions by the Wiener's measure.) However, assuming that we can give a meaning to the path integrals, this formulation allows to recover the classical values, for instance $\langle\phi(x)\rangle \sim \phi(x)$, when $\hbar \rightarrow 0$.

5.3 - Free fields. The quantization of a classical free field $\phi_{0}$ is easy. In fact, the action $S_{0}\left[\phi_{0}\right]=\frac{1}{2} \int d^{D} x \phi_{0}(x) A \phi_{0}(x)$ is quadratic in $\phi_{0}$ and gives rise to a Gaussian measure, $\exp \left(-\frac{S\left[\phi_{0}\right]}{\hbar}\right) d \phi_{0}$. If the field is isolated, the Green's functions are then easily computed:

- the mean value $\left\langle\phi_{0}(x)\right\rangle$ is zero;

- the 2 points Green's function $\left\langle\phi_{0}(x) \phi_{0}(y)\right\rangle$ coincides with the Green's function $G_{0}(x-y)$;

- all the Green's functions on an odd number of points are zero;

- the Green's functions on an even number of points are products of Green's functions exhausting all the points.

If the field is not isolated, instead, as well as when the field is self-interacting, the computation of the Green's functions are more involved.

5.4 - Dyson-Schwinger equation. In general, the Green's functions satisfy an integro-differential equation which generalises the Euler-Lagrange equation, written in the form $\frac{\partial S[\phi]}{\partial \phi(x)}=0$. To obtain this equation, in analogy with the analysis that one would perform on a finite dimensional set of paths, one can proceed by introducing a generating functional for Green's functions. The self-standing of the results is considered sufficient to accept the intermediate meaningless steps.

To do it, let us regard the action as a function also of the classical source field $J$, that is $S[\phi]=S[\phi, J]$. Then we define the partition function

$$
Z[J]=\int d \phi e^{-\frac{S[\phi]}{\hbar}}
$$

and we impose the normalization condition $\left.Z[J]\right|_{J=0}=1$. It is then easy to verify that the Green's functions can be derived from the partition function, as

$$
\left\langle\phi\left(x_{1}\right) \cdots \phi\left(x_{k}\right)\right\rangle=\left.\frac{\hbar^{k}}{Z[J]} \frac{\delta^{k} Z[J]}{\delta J\left(x_{1}\right) \cdots \delta J\left(x_{k}\right)}\right|_{J=0} .
$$

The Dyson-Schwinger equation for Green's functions, then, can be deduced from a functional equation which constrains the partition function:

$$
\frac{\delta S}{\delta \phi(x)}\left[\hbar \frac{\delta}{\delta J}\right] Z[J]=0 .
$$

The notation used on the left hand-side means that in the functional $\frac{\delta S}{\delta \phi(x)}$ of $\phi$, we substitute the variable $\phi$ with the operator $\hbar \frac{\delta}{\delta J}$. Since $S[\phi]$ is a poynomial, we obtain an operator which contains some repeted derivations with respect to $J$, and which can then act on $Z[J]$.

\footnotetext{
${ }^{1}$ On the Minkowski space this value is $\exp \left(i \frac{S[\phi]}{\hbar}\right)$.
} 
5.5 - Connected Green's functions. If, starting from the partition function, we define the free energy

$$
W[J]=\hbar \ln Z[J], \quad \text { i.e. } \quad Z[J]=e^{\frac{W[J]}{\hbar}},
$$

with normalization condition $\left.W[J]\right|_{J=0}=0$, we see that the correlation functions are sums of recursive terms (products of Green's functions on a smaller number of points), and additional terms which involve the derivatives of the free energy:

$$
\begin{aligned}
\langle\phi(x)\rangle & =\left.\frac{\hbar}{Z[J]} \frac{\delta Z[J]}{\delta J(x)}\right|_{J=0}=\left.\frac{\delta W[J]}{\delta J(x)}\right|_{J=0}, \\
\langle\phi(x) \phi(y)\rangle & =\langle\phi(x)\rangle\langle\phi(y)\rangle+\left.\hbar \frac{\delta^{2} W[J]}{\delta J(x) \delta J(y)}\right|_{J=0}, \ldots
\end{aligned}
$$

These additional terms

$$
G\left(x_{1}, \ldots, x_{k}\right)=\left.\frac{\delta^{k} W[J]}{\delta J\left(x_{1}\right) \cdots \delta J\left(x_{k}\right)}\right|_{J=0}
$$

are called connected Green's functions, for reasons which will be clear after we introduced the Feynman diagrams. Of course, knowing the connected Green's functions $G\left(x_{1}, \ldots, x_{k}\right)$ is enough to recover the full Green's functions $\left\langle\phi\left(x_{1}\right) \cdots \phi\left(x_{k}\right)\right\rangle$, through the relations:

$$
\begin{aligned}
\langle\phi(x)\rangle=G(x) & \\
\langle\phi(x) \phi(y)\rangle=G(x) & G(y)+\hbar G(x, y) \\
\langle\phi(x) \phi(y) \phi(z)\rangle=G(x) & G(y) G(z)+\hbar[G(x) G(y, z)+G(y) G(x, z)+G(z) G(x, y)]+\hbar^{2} G(x, y, z), \\
\langle\phi(x) \phi(y) \phi(z) \phi(u)\rangle=G(x) & G(y) G(z) G(u) \\
& +\hbar[G(x) G(y) G(z, u)+\text { terms }] \\
& +\hbar^{2}[G(x, y) G(z, u)+\text { terms }+G(x) G(y, z, u)+\text { terms }] \\
& +\hbar^{3} G(x, y, z, u)
\end{aligned}
$$

and so on, where by "terms" we mean the same products evaluated on suitable permutations of the points $(x, y, z, u)$.

5.6 - Self-interacting fields. The Dyson-Schwinger equation can be expressed in terms of the connected Green's functions. To be precise, we consider the typical quantum field with classical action

$$
S[\phi]=\frac{1}{2} \phi^{t} A \phi-J^{t} \phi-\frac{\lambda}{3 !} \int d^{D} x \phi(x)^{3},
$$

and we denote by $G_{0}=A^{-1}$ the resolvent of the operator $A$. Then, the Dyson-Schwinger equation for the 1-point correlation function of a field in an external field $J$ is

$$
\langle\phi(x)\rangle_{J}=\frac{\delta W[J]}{\delta J(x)}=\int d^{D} u G_{0}(x-u)\left[J(u)+\frac{\lambda}{2}\left[\left(\frac{\delta W[J]}{\delta J(u)}\right)^{2}+\hbar \frac{\delta^{2} W[J]}{\delta J(u)^{2}}\right]\right] .
$$

If we evaluate Eq. (5.6.1) at $J=0$, we obtain the Dyson-Schwinger equation for the 1-point Green's function of an isolated field:

$$
\langle\phi(x)\rangle=G(x)=\frac{\lambda}{2} \int d^{D} u G_{0}(x-u)\left[G(u)^{2}+\hbar G(u, u)\right] .
$$

If we derive Eq. (5.6.1) by $\frac{\delta}{\delta J(y)}$, and evaluate at $J=0$, we obtain the Dyson-Schwinger equation for the 2-points connected correlation function:

$$
G(x, y)=G_{0}(x-y)+\frac{\lambda}{2} \int d^{D} u G_{0}(x-u)[2 G(u) G(u, y)+\hbar G(u, u, y)]
$$


which involves the 3-points Green's function. Repeating the derivation, we get the Dyson-Schwinger equation for the n-points connected Green's function.

As for classical interacting fields, these equations can be solved perturbatively. For instance, the solution of Eq. (5.6.1), that is the mean value of a field $\phi$ in an external field $J$, is:

$$
\begin{aligned}
\langle\phi(x)\rangle_{J} & =\int d^{D} u G_{0}(x-u) J(u) \\
& +\frac{\lambda}{2} \int d^{D} y d^{D} z d^{D} u G_{0}(x-y) G_{0}(y-z) G_{0}(y-u) J(z) J(u) \\
& +\frac{2 \lambda^{2}}{4} \int d^{D} y d^{D} z d^{D} u d^{D} v d^{D} w G_{0}(x-y) G_{0}(y-z) G_{0}(y-u) G_{0}(z-v) G_{0}(z-w) \\
& \quad \times J(z) J(u) J(v) J(w) \\
& +\hbar \frac{\lambda}{2} \int d^{D} y G_{0}(x-y) G_{0}(y-y) \\
& +\hbar \frac{\lambda^{2}}{2} \int d^{D} y d^{D} z d^{D} u G_{0}(x-y) G_{0}(y-z)^{2} G_{0}(z-u) J(u)+\mathcal{O}\left(\lambda^{3}\right) .
\end{aligned}
$$

Of course, the mean value of the isolated field, that is the solution of Eq. (5.6.2), is then obtained by setting $J=0$ :

$$
G(x)=\hbar \frac{\lambda}{2} \int d^{D} y G_{0}(x-y) G_{0}(y-y)+\mathcal{O}\left(\lambda^{3}\right) .
$$

5.7 - Exercise: 2-points connected Green's function. Compute the first perturbative terms of the solution of Eq. (5.6.3), which represents the Green's function $G(x, y)$ for an isolated field $(J=0)$.

5.8 - Conclusion. For a typical quantum field $\phi$ with classical Lagrangian density of the form

$$
\mathcal{L}(\phi)=\frac{1}{2} \phi^{t} A \phi-\frac{\lambda}{3 !} \phi(x)^{3},
$$

- the full $k$-points Green's function $\left\langle\phi\left(x_{1}\right) \cdots \phi\left(x_{k}\right)\right\rangle$ is the sum of the products of the connected Green's functions exhausting the $k$ external points;

- the connected $k$-points Green's function can be described perturbatively as a formal series

$$
G\left(x_{1}, \ldots, x_{k}\right)=\sum_{n=0}^{\infty} \lambda^{n} G_{n}\left(x_{1}, \ldots, x_{k}\right)
$$

in the powers of the coupling constant $\lambda$;

- the constant coefficient $G_{0}\left(x_{1}, \ldots, x_{k}\right)$ is the Green's function of the free field;

- each higher order coefficient $G_{n}\left(x_{1}, \ldots, x_{k}\right)$ is a finite sum of integrals involving only the free propagator.

We describe the sums appearing in $G_{n}\left(x_{1}, \ldots, x_{k}\right)$ in Lecture III using Feynman graphs.

\section{Lecture III - Formal series expanded over Feynman graphs}

In this lecture we consider a quantum field $\phi$ with classical Lagrangian density of the form

$$
\mathcal{L}(\phi)=\frac{1}{2} \phi^{t} A \phi-J(x) \phi(x)-\frac{\lambda}{3 !} \phi(x)^{3},
$$

where $A$ is a differential operator, typically the Klein-Gordon operator. We denote by $G_{0}$ the Green's function of $A$. We saw in Section 5 that the Green's functions of this field are completely determined by the connected Green's functions, and that these can only be described as formal series in the powers of the coupling constant,

$$
G\left(x_{1}, \ldots, x_{k}\right)=\sum_{n=0}^{\infty} \lambda^{n} G_{n}\left(x_{1}, \ldots, x_{k}\right) .
$$

In this section we describe the coefficients $G_{n}\left(x_{1}, \ldots, x_{k}\right)$ using Feynman diagrams. We begin by describing the coefficients of the perturbative solution $\phi(x)=\sum \lambda^{n} \phi_{n}(x)$ for the classical field. 


\section{Interacting classical fields}

6.1 - Feynman notations. We adopt the following Feynman's notations for the field $\phi$ :

- field $\phi(x)=x \bullet \oslash$;

- source $J(y)=\longrightarrow x^{y}$;

- propagator $G_{0}(x-y)=x \longmapsto y$.

For each graphical object resulting from Feynman's notation, we call amplitude its analytical value.

6.2 - Euler-Lagrange equation. The Euler-Lagrange equation (4.6.1) is represented by the following diagrammatic equation:

$$
x \longmapsto \varnothing=x \longmapsto x^{y}+\frac{\lambda}{2} x \longmapsto
$$

6.3 - Perturbative expansion on trees. Inserting the value of $y \_-$on the right hand-side of Eq. 6.2.1, and repeating the insertion until all the black boxes have disappeared on the right hand-side, we obtain a perturbative solution given by a formal series expanded on trees, which are graphs without loops in the space:

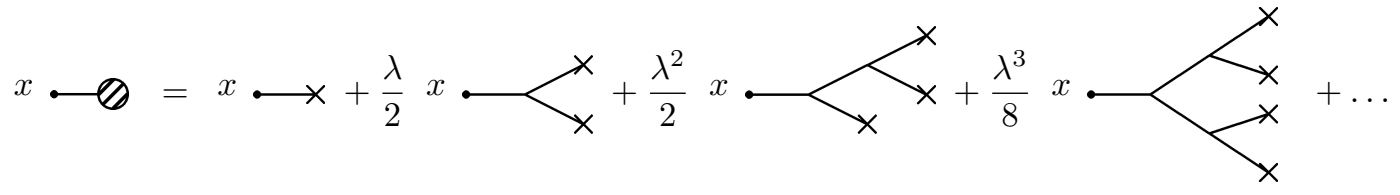

The coefficient of each tree $t$ contains a factor $\lambda^{V(t)}$ where $V(t)$ is the number of internal vertices of the tree, and at the denominator the symmetry factor $\operatorname{Sym}(t)$ of the tree, that is the number of permutations of the external crosses (the sources) which leave the tree invariant.

If we compare the diagrammatic solution (6.3.1) with the explicit solution (4.6.2), we can write explicitely the value $\phi_{t}(x)$ of each tree $t$, for instance:

$$
\begin{aligned}
& t=\longleftrightarrow \times \Longrightarrow \phi_{t}(x)=\int d^{D} y G_{0}(x-y) J(y) \quad, \\
& t=<_{\times}^{\times} \Longrightarrow \phi_{t}(x)=\int d^{D} y d^{D} z d^{D} u G_{0}(x-y) G_{0}(y-z) G_{0}(y-u) J(z) J(u) .
\end{aligned}
$$

Finally note that the valence of the internal vertices of the trees depends directly on the interacting term of the Lagrangian. In the above example this term was $-\frac{\lambda}{3 !} \phi^{3}$. If the Lagrangian contains the interacting term $-\frac{\mu}{4 !} \phi^{4}$, the internal vertices of the trees turn out to have valence 4 , that is, the trees are of the form

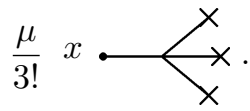

6.4 - Feynman rules. We can therefore conclude that the field $\phi(x)=\sum_{n} \lambda^{n} \phi_{n}(x)$ has perturbative coefficients $\phi_{n}(x)$ given by the finite sum of the amplitude $\phi_{t}(x)$ of all the trees $t$ with $n$ internal vertices, constructed according to the following Feynman's rules:

- consider all the trees with internal vertices of valence 3 , and external vertices of valence 1 ;

- fix one external vertex called the root (therefore the trees are called rooted), and call the other external vertices the leaves;

- label the root by $x$;

- label the internal vertices and the leaves by free variables $y, z, u, v, \ldots$;

- assign a weigth $G_{0}(y-z)$ to each edge joining the vertices $y$ and $z$; 
- assign a weigth $\lambda$ to each internal vertex $<$;

- assign a weigth $J(y)$ to each leaf;

- to obtain $\phi_{t}(x)$ for a given tree $t$, multiply all the weigths and integrate over the free variables;

- divide by the symmetry factor $\operatorname{Sym}(t)$ of the tree.

6.5 - Conclusion. A typical classical field $\phi$ with Lagrangian density of the form

$$
\mathcal{L}(\phi)=\frac{1}{2} \phi^{t} A \phi-J(x) \phi(x)-\frac{\lambda}{3 !} \phi(x)^{3},
$$

can be described as a formal series in the coupling constant $\lambda$,

$$
\phi(x)=\sum_{n=0}^{\infty} \lambda^{n} \phi_{n}(x),
$$

where each coefficient $\phi_{n}(x)$ is a finite sum

$$
\phi_{n}(x)=\sum_{V(t)=n} \frac{1}{\operatorname{Sym}(t)} \phi_{t}(x)
$$

of amplitudes $\frac{1}{\operatorname{Sym}(t)} \phi_{t}(x)$ associated to each tree $t$ with $n$ internal vertices of valence 3 . Note that, in these lectures, the amplitude of a tree is considered modulo the factor $\frac{1}{\operatorname{Sym}(t)}$.

\section{$7 \quad$ Interacting quantum fields}

7.1 - Feynman notations. We adopt the following Feynman's notations:

- $k$-points full Green's function $\left\langle\phi\left(x_{1}\right) \cdots \phi\left(x_{k}\right)\right\rangle=x_{k}$

- $k$-points connected Green's function $G\left(x_{1}, \ldots, x_{k}\right)=$

- source $J(y)=\longrightarrow x^{y}$;

- propagator $G_{0}(x-y)=x \longmapsto y$.

7.2 - Exercise: Diagrammatic expression of the full Green's functions. Using Eqs. (5.5.1), draw the diagrammatic expression of the full Green's functions in terms of the connected ones.

7.3 - Dyson-Schwinger equations. The Dyson-Schwinger equation for the 1-point connected Green's function of a field in presence of an external field $J$ (cf. Eq. (5.6.1)), is the following:

$$
x \longmapsto \varnothing=x \longmapsto+\frac{\lambda}{2} x \longmapsto
$$

Note that in the limit $\hbar \rightarrow 0$, we recover the Euler-Lagrange equation (6.2.1) for the field.

The Dyson-Schwinger equation for the 1-point connected Green's function of an isolated field (cf. Eq. (5.6.2)), is the following:

$$
x \smile \emptyset=\frac{\lambda}{2} x \multimap
$$


For the 2-points connected Green's function, the Dyson-Schwinger equation is (cf. Eq. (5.6.3)):

$$
x \longmapsto y=x \longmapsto y+\lambda x \longmapsto y+\hbar \frac{\lambda}{2} x \curvearrowleft y .
$$

For the 3-points Green's function:

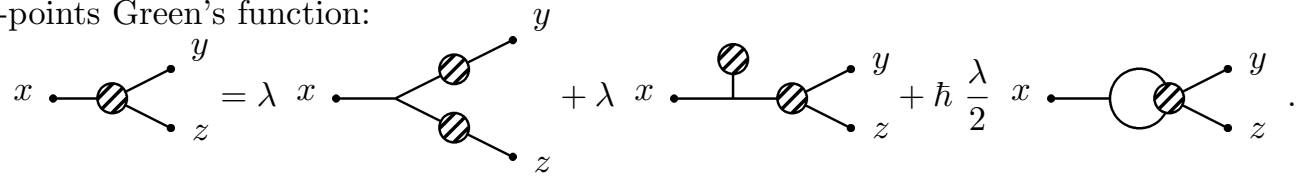

7.4 - Perturbative expansion on graphs. Then the perturbative solution of the Dyson-Schwinger equation is given by a formal series expanded on Feynman diagrams, which are graphs in the space. For the 1-point Green's function, the solution of (7.3.1) is $(J \neq 0)$ :

$$
=\longrightarrow x+\frac{\lambda}{2}
$$

The coefficient of each graph $\Gamma$ contains a factor $\lambda^{V}(\Gamma)$ where $V(\Gamma)$ is the number of internal vertices of the graph, and at the denominator the symmetry factor $\operatorname{Sym}(\Gamma)$ of the graph, that is the number of permutations of the external crosses (the sources) and of internal edges (those which are not Joint to external vertex) which leave the graph invariant.

Of course, the solution of Eq. (7.3.2) is $(J=0)$ :

$$
\smile=\hbar \frac{\lambda}{2} \multimap 0+\hbar^{2} \frac{\lambda^{3}}{4} \multimap \bigcirc+\hbar^{2} \frac{\lambda^{3}}{4} \curvearrowleft \bigcirc+\mathcal{O}\left(\lambda^{4}\right) \text {. }
$$

For the 2-points Green's function, the solution of Eq. (7.3.3) is

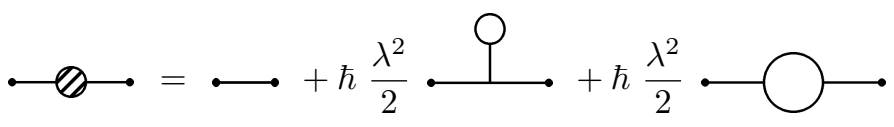

$$
\begin{aligned}
& +\hbar^{2} \frac{\lambda^{4}}{4} \multimap \bigcirc+\hbar^{2} \frac{\lambda^{4}}{2} \multimap \bigcirc
\end{aligned}
$$

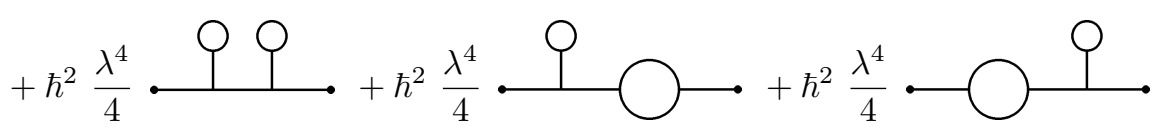

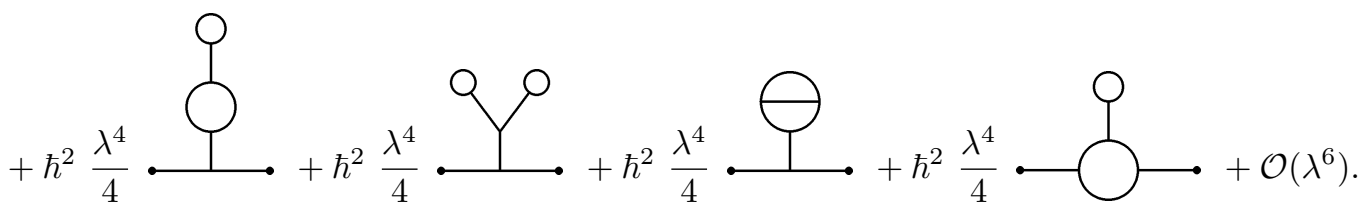

Note that the grey boxes contain all the connected graphs. This motivates the name of the connected Green's functions.

7.5 - Exercise: 3-points connected Green's function. Write the diagrammatic expansion of the 3-points connected correlation function, that is the solution of Eq. (7.3.4).

7.6 - Feynman rules. We can therefore conclude that each connected Green's function $G\left(x_{1}, \ldots, x_{k}\right)=$ $\sum_{n} \lambda^{n} G_{n}\left(x_{1}, \ldots, x_{k}\right)$ has perturbative coefficients $G_{n}\left(x_{1}, \ldots, x_{k}\right)$ given by the finite sum of the amplitude $A\left(\Gamma ; x_{1}, \ldots, x_{k}\right)$ of all the Feynman graphs with $n$ internal vertices, constructed according to the following Feynman's rules (valid for $J=0$ ): 
- consider all the graphs with internal vertices of valence 3 , and $k$ external vertices of valence 1 ;

- label the external vertices by $x_{1}, \ldots, x_{k}$;

- label the internal vertices by free variables $y, z, u, v, \ldots$;

- assign a weigth $G_{0}(y-z)$ to each edge joining the vertices $y$ and $z$;

- assign a weigth $\lambda$ to each internal vertex $<$;

- assign a weigth $\hbar$ to each loop

- to obtain $A\left(\Gamma ; x_{1}, \ldots x_{k}\right)$ for a given graph $\Gamma$, multiply all the weigths and integrate over the free variables;

- divide by the symmetry factor $\operatorname{Sym}(\Gamma)$ of the graph.

7.7 - Exercise: Feynman's rules in presence of an external source. Modify the Feynman's rules given above so that they are valid when $J \neq 0$.

7.8 - Exercise: compute some amplitudes. Compute the amplitudes of the first Feynman graphs appearing in the expansions of the 2-points Green's function given above, using the Feynman's rules, and compare them with the results in Exercise 5.7.

7.9 - Conclusion. For a typical quantum field $\phi$ with Lagrangian density of the form

$$
\mathcal{L}(\phi)=\frac{1}{2} \phi^{t} A \phi-J(x) \phi(x)-\frac{\lambda}{3 !} \phi(x)^{3},
$$

the connected $k$-points Green's function can be described as a formal series

$$
G\left(x_{1}, \ldots, x_{k}\right)=\sum_{n=0}^{\infty} \lambda^{n} G_{n}\left(x_{1}, \ldots, x_{k}\right),
$$

where each coefficient $G_{n}\left(x_{1}, \ldots, x_{k}\right)$ is a finite sum

$$
G_{n}\left(x_{1}, \ldots, x_{k}\right)=\sum_{V(\Gamma)=n} \frac{\hbar^{L(\Gamma)}}{\operatorname{Sym}(\Gamma)} A\left(\Gamma ; x_{1}, \ldots, x_{k}\right)
$$

of amplitudes $A\left(\Gamma ; x_{1}, \ldots, x_{k}\right)$ associated to each connected Feynman diagram $\Gamma$ with $n$ internal vertices of valence 3. Note that, in these lectures, the amplitude of a graph is considered modulo the factor $\frac{\hbar^{L(\Gamma)}}{\operatorname{Sym}(\Gamma)}$.

\section{Field theory on the momentum space}

8.1 - Momentum coordinates. In relativistic quantum mechanics, the four-momentum $p$, that we call simply momentum here, is the conjugate variable of the four-position $x$, seen as an operator of multiplication on the wave function. Therefore the momentum is the Fourier transform of the operator of derivation by the position, and belongs to the Fourier space $\widehat{\mathbb{R}^{D}}$.

To express the field theory on the momentum variables, we Fourier transform all the components of the equation of motion:

$$
\begin{aligned}
\widehat{\phi}(p) & =\int_{\mathbb{R}^{D}} d^{D} x \phi(x) e^{i p \cdot x}, \\
\widehat{J}(p) & =\int_{\mathbb{R}^{D}} d^{D} x J(x) e^{i p \cdot x}, \\
\widehat{G_{0}}(p) & =\int_{\mathbb{R}^{D}} d^{D} x G_{0}(x-y) e^{i p \cdot(x-y)},
\end{aligned}
$$


for instance, for the Klein-Gordon field, $\widehat{G_{0}}(p)=\frac{1}{p^{2}+m^{2}}$ is the Fourier transform of the free propagator (4.5.2). The classical Euler-Lagrange equation (4.6.1) is then transformed into

$$
\widehat{\phi}(p)=\widehat{G_{0}}(p) \widehat{J}(p)+\frac{\lambda}{2} \widehat{G_{0}}(p) \int \frac{d^{D} q}{(2 \pi)^{D}} \widehat{\phi}(q) \widehat{\phi}(p-q) .
$$

The Fourier transform of the Green's functions is

$$
\widehat{G}^{(k)}\left(p_{1}, \ldots, p_{k}\right)=\int_{\left(\mathbb{R}^{D}\right)^{k}} d^{D} x_{1} \ldots d^{D} x_{k} G\left(x_{1}, \ldots, x_{k}\right) e^{i p_{1} \cdot\left(x_{1}-x_{k}\right)} \cdots e^{i p_{k} \cdot\left(x_{k-1}-x_{k}\right)},
$$

where the translation invariance of $G\left(x_{1}, \ldots, x_{k}\right)$ implies that $\sum_{i=1, \ldots, k} p_{i}=0$, and the Dyson-Schwinger equations (5.6.2), (5.6.3), etc, can easily be expressed in terms of external momenta:

$$
\begin{aligned}
& \widehat{G}^{(1)}(0)=\frac{\lambda}{2} \widehat{G_{0}}(0)\left(\widehat{G}^{(1)}(0)\right)^{2}+\hbar \frac{\lambda}{2} \int \frac{d^{D} q}{(2 \pi)^{D}} \widehat{G}^{(2)}(q), \\
& \widehat{G}^{(2)}(p)=\widehat{G_{0}}(p)+\lambda \widehat{G_{0}}(p) \widehat{G}^{(1)}(0) \widehat{G}^{(2)}(p)+\hbar \frac{\lambda}{2} \widehat{G_{0}}(p) \int \frac{d^{D} q}{(2 \pi)^{D}} \widehat{G}^{(3)}(q, p-q,-p),
\end{aligned}
$$

and so on.

8.2 - Feynman graphs on the momentum space. The Feynman graphs on the momentum space look exactely like those on the space-time coordinates, except that the external legs are not fixed in the dotted positions $x_{1}, \ldots, x_{k}$, but have oriented edges, and in particular oriented external legs labeled by momenta $p_{1}, \ldots, p_{k}$, where the arrows tell what is the direction of the propagation. The Feynman notations are:

- field $\widehat{\phi}(p)=\rightarrow \underset{p}{ }$, or $k$-points connected Green's function $\widehat{G}^{(k)}\left(p_{1}, \ldots, p_{k}\right)=\stackrel{p_{1}}{p_{k}}$

- propagator $\widehat{G_{0}}(p)=\vec{p}$;

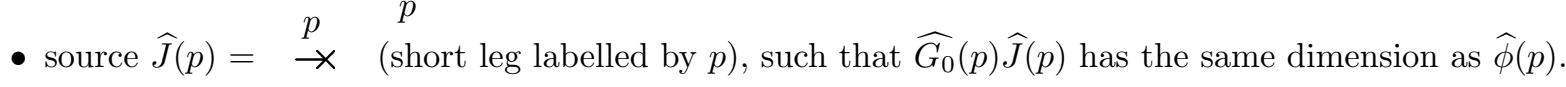

The Feynman graphs with short external legs are sometimes called truncated or amputated. Modulo these few differences, the Euler-Lagrange equation, the Dyson-Schwinger equations, and their perturbative solutions, are the same as those already given on the space-time coordinates.

To simplify the notations, from now on we denote by $G_{0}(p)$ the free propagator also in the momentum space, instead of $\widehat{G_{0}}(p)$, and in general we omit the hat symbol. Similarly, we omit the orientation of the propagators unless necessary.

8.3 - One-particle irreducible graphs. The Feynman rules, which allow us to write the amplitude of a Feynman graph, implicetely state that the amplitude of a non-connected graph is the product of the amplitudes of all its connected components (cf. Eqs. (5.5.1) and Exercise 7.2). If we work in the momentum space, then from the Feynman rules it also follows that if a graph $\Gamma$ is the junction of two subgraphs $\Gamma_{1}$ and $\Gamma_{2}$, through a simple edge, that is

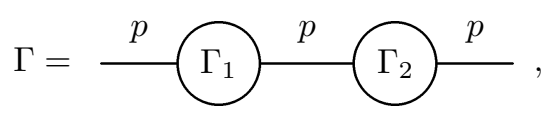

then the amplitude of $\Gamma$ is the product of the amplitude of the single graphs, that is

$$
A(\Gamma ; p)=G_{0}(p) A\left(\Gamma_{1} ; p\right) G_{0}(p) A\left(\Gamma_{2} ; p\right) G_{0}(p),
$$

where $\Gamma_{1}$ and $\Gamma_{2}$ here are truncated on both sides. (Note that the internal edge must have momentum $p$ because of the conservation of total momentum at each vertex.) 
We say that a connected Feynman graph $\Gamma$ is one-particle irreducible, in short 1PI, if it remains connected when we cut one of its edges. In particular, the free propagator — is not 1PI, therefore the 1PI graphs in the momentum space are truncated. For instance, the graphs

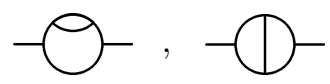

are 1PI, while the graphs

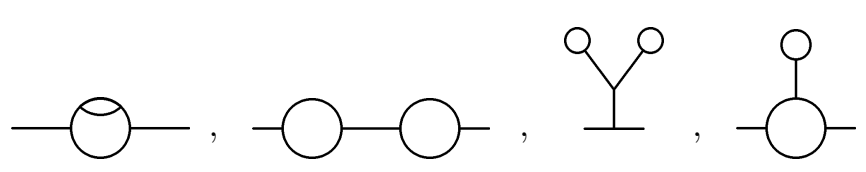

are not 1PI. If we denote the junction of graphs through one of their external legs by the concatenation, for instance

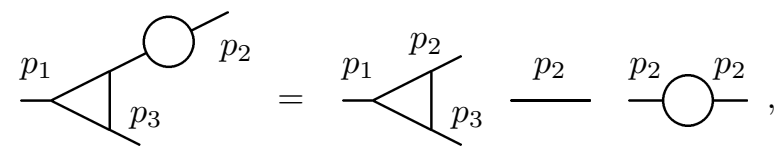

then any connected graph can then be seen as the concatenantion of its 1PI components and the free propagators necessary to joint them. To avoid these free propagators popping out at any cut, we can consider graphs which are truncated only on some of their external legs, and allow to joint truncated legs with full ones, for instance

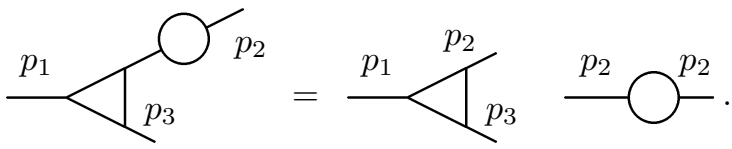

With this trick, any connected graph $\Gamma$ can be seen as the junction $\Gamma=\Gamma_{1} \cdots \Gamma_{s}$ of its 1PI components (modulo some free propagators).

8.4 - Proper or 1PI Green's functions. The fact that any connected Feynman graph can be reconstructed from its 1PI components implies that the connected Green's function

$$
G^{(k)}\left(p_{1}, \ldots, p_{k}\right)=\sum_{E(\Gamma)=k} \lambda^{V(\Gamma)} \frac{\hbar^{L(\Gamma)}}{\operatorname{Sym}(\Gamma)} A\left(\Gamma ; p_{1}, \ldots, p_{k}\right),
$$

where the sum is over all connected graphs with $k$ external legs, can be reconstructed from the set of proper or 1 PI Green's functions

$$
G_{1 \mathrm{PI}}^{(k)}\left(p_{1}, \ldots, p_{k}\right)=\sum_{\substack{E(\Gamma)=k \\ 1 \mathrm{PI} \Gamma}} \lambda^{V(\Gamma)} \frac{\hbar^{L(\Gamma)}}{\operatorname{Sym}(\Gamma)} A\left(\Gamma ; p_{1}, \ldots, p_{k}\right),
$$

where the sum is now over 1PI graphs suitably truncated. The precise relation between connected and proper Green's functions can be given easily only for the 2-point Green's functions: in this case we have

$$
G^{(2)}(p)=.
$$

The general case is much more involved, and was treated recently using algebraic tools by $\hat{A}$. Mestre and R. Oeckl in [21].

8.5 - Conclusion. In summery, for a typical quantum field $\phi$ with Lagrangian density of the form

$$
\mathcal{L}(\phi)=\frac{1}{2} \phi^{t} A \phi-J(x) \phi(x)-\frac{\lambda}{3 !} \phi(x)^{3},
$$

the connected $k$-points Green's function on the momentum space can be described as a formal series

$$
G\left(p_{1}, \ldots, p_{k}\right)=\sum_{n=0}^{\infty} \lambda^{n} G_{n}\left(p_{1}, \ldots, p_{k}\right)
$$


where each coefficient $G_{n}\left(p_{1}, \ldots, p_{k}\right)$ is a finite sum of amplitudes associated to each (partially amputated) connected Feynman diagram with $n$ internal vertices of valence 3, and the amplitude of each graph $\Gamma$ is the product of the amplitudes of its 1 PI components $\Gamma_{i}$, that is

$$
\begin{aligned}
G_{n}\left(p_{1}, \ldots, p_{k}\right) & =\sum_{V(\Gamma)=n} \frac{\hbar^{L(\Gamma)}}{\operatorname{Sym}(\Gamma)} A\left(\Gamma ; p_{1}, \ldots, p_{k}\right) \\
& =\sum_{V(\Gamma)=n} \prod_{\Gamma=\Gamma_{1} \cdots \Gamma_{s}} \frac{\hbar^{L\left(\Gamma_{i}\right)}}{\operatorname{Sym}\left(\Gamma_{i}\right)} A\left(\Gamma_{i} ; p_{1}^{(i)}, \ldots, p_{k_{i}}^{(i)}\right) .
\end{aligned}
$$

\section{Lecture IV - Renormalization}

In Lecture II we computed the first terms of the perturbative solution of the classical and the quantum interacting fields. As we saw in Lecture III, these terms can be regarded as the amplitudes of some useful combinatorial objects, the rooted trees and the Feynman's graphs. These analitic expressions, the amplitudes, are constructed as repeated integrals of products of the field propagator $G_{0}$ and eventually an external field $J$. The field propagator $G_{0}(x)$ is a distribution of the point $x$, and it is singular in $x=0$ if $n>1$. Then, the square $G_{0}(x)^{2}$ is a continuous function for $x \neq 0$, but it is not defined in $x=0$. On the momentum space, this problem is translated into the divergency of the integral containing powers of the free propagator.

The powers of a free propagator never occur in the amplitude of the trees labelling the perturbative expansion of classical fields, cf. Eq. (4.6.2). Similarly, they do not occur in the classical part of the perturbative expansion of Green's functions for a quantum field (that is, those terms which are not factors of $\hbar$ ). Instead, such terms occur in the quantum corrections, that is, the terms which are factors of $\hbar$. For instance, the last two terms in Eq. (5.6.4) contain $G_{0}(y-y)=G_{0}(0)$ and the square $G_{0}(y-z)^{2}$ which is meaningless for $y=z$.

In this lecture we explain some tools developped to give a meaning to the ill-defined terms appearing in the perturbative expansions of the Green's functions. This technique is known as the theory of renormalization.

\section{Renormalization of Feynman amplitudes}

The renormalization of the ill-defined amplitudes can be done for graphs on the momentum variables as well as on the space-time variables. On the space-time variables, the renormalization program has been described by H. Epstein and V. J. Glaser in [12], in the context of the causal perturbation theory. However, to describe renormalization, it is convenient to work on the momentum space and to consider 1PI graphs.

9.1 - Problem of divergent integrals: ultraviolet and infrared divergencies. In dimension $D=1$, all the integrals appearing in the perturbative expansion of the Green's functions are convergent. For example, if we consider the Klein-Gordon field $\phi$, the free propagator

$$
G_{0}(x-y)=\int_{\mathbb{R}} \frac{d p}{2 \pi} \frac{e^{-i p(x-y)}}{p^{2}+m^{2}}
$$

is a continuous function. Therefore all the products of propagators are also continuous functions, and the integrals are well defined.

In dimension $D>1$, the free propagator $G_{0}(x-y)$ is a singular distribution on the diagonal $x=y$, and the product with other distributions which are singular at the same points, such as its powers $G_{0}(x-y)^{m}$, makes no sense. For the Klein-Gordon field, for example, this happens already in the simple loop

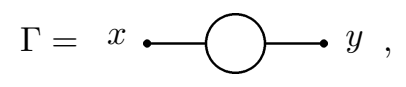

whose amplitude

$$
A(\Gamma ; x, y)=\int d^{D} u d^{D} v G_{0}(x-u) G_{0}(u-v)^{2} G_{0}(v-z)
$$


contains the square $G_{0}(u-v)^{2}$. To understand how the integral is affected by the singularity, we better write the simple loop on the momentum space. The Fourier transform of $\Gamma$ gives the (truncated) simple loop

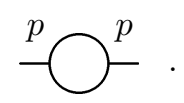

To compute its amplitude, we write the integrated momentum $q$ in spherical coordinates, with $|q|$ denoting the module. Then we see that for $|q| \rightarrow \infty$ the integral

$$
\int \frac{d^{D} q}{(2 \pi)^{D}} \frac{1}{q^{2}+m^{2}} \frac{1}{(p-q)^{2}+m^{2}}
$$

roughly behaves like

$$
\int_{|q|_{\text {min }}}^{\infty} d|q|^{D} \frac{1}{|q|^{4}} \simeq \int_{|q|_{\text {min }}}^{\infty} d|q| \frac{1}{|q|^{4-(D-1)}} .
$$

This integral converges if and only if $4-(D-1)>1$, that is $D<4$. Therefore $A(\Gamma ; x, y)$ diverges when the dimension of the base-space is $D \geq 4$.

The divergency of an amplitude $A(\Gamma ; p)$ which occurs when an integrated variable $q$ has module $|q| \rightarrow \infty$ is called ultraviolet. The divergency which occurs when $|q| \rightarrow|q|_{\min }$ is called infrared. The infrared divergencies appear typically when the mass $m$ is zero and $|q|_{\min }=0$ (for instance, for photons). In this lecture we only deal with ultraviolet divergencies.

To simplify the notations, if $\Gamma$ is a graph with $k$ external legs, we denote its amplitudes $A\left(\Gamma ; x_{1}, \ldots, x_{k}\right)$ or $A\left(\Gamma ; p_{1}, \ldots, p_{k}\right)$ simply by $A(\Gamma)$, when the dependence on the external parameters $x_{1}, \ldots, x_{k}$ or $p_{1}, \ldots, p_{k}$ is not relevant.

9.2 - Renormalized amplitudes, normalization conditions and renormalisable theories. There is a general procedure to estimate which integrals are divergent, and then to extract from each infinite value a finite contribution which has a physical meaning. This program is called the renormalization of the amplitude of Feynman graphs.

Given a graph $\Gamma$ with divergent amplitude $A(\Gamma)$, the aim of the renormalization program is to find a finite contribution $A^{\text {ren }}(\Gamma)$, called renormalized amplitude, which satisfies some physical requirements. In contraposition to the renormalized amplitude, the original divergent amplitude is often called bare or nude.

The physical conditions required, called normalization conditions, are those which guarantee that the connected Green's function and its derivatives have a precise value at a given point. The theory is called renormalisable if the number of conditions that we have to impose to determine the amplitude of all Feynaman graphs is finite. For instance, the $\phi^{3}$ theory is renormalizable in dimension $D \leq 6$.

9.3 - Power counting: classification of one loop divergencies. The superficial degree of divergency of a 1PI graph $\Gamma$ measures the degree of singularity $\omega(\Gamma)$ of the integral in $A(\Gamma)$ with respect to the integrated variables $q_{1}, q_{2}, \ldots$. By definition, $\omega(\Gamma)$ is the integer such that, under the transformation of momentum $q_{i} \rightarrow t q_{i}$, with $t \in \mathbb{R}$, the amplitude is transformed as

$$
A(\Gamma) \quad \longrightarrow \quad t^{\omega(\Gamma)} A(\Gamma)
$$

The superficial degree of divergency detects the "real" divergency only for diagrams with one single loop: in this case $A(\Gamma)$ converges if and only if $\omega(\Gamma)$ is negative. The divergencies for single-loop graphs are then classified according to $\omega(\Gamma)$ :

- a graph $\Gamma$ has a logarithmic divergency if $\omega(\Gamma)=0$;

- it has a polynomial divergency of degree $\omega(\Gamma)$ if $\omega(\Gamma)>0$. That is, the divergency is linear if $\omega(\Gamma)=1$, it is quadratic if $\omega(\Gamma)=2$, and so on.

If the graph contains many loops, instead, it can have a negative value of $\omega(\Gamma)$ and at the same time contain some divergent subgraphs. Therefore $\omega(\Gamma)$ can not be used to estimate the real (not superficial) divergency of a graph $\Gamma$ with many loops. In this case, we first have to compute $\omega(\gamma)$ for each single 1PI subgraph $\gamma$ of 
$\Gamma$, starting from the subgraphs with a simple loop and proceding by enlarging the subgraphs until we reach $\Gamma$ itself. This recursive procedure on the subgraphs will be discussed in details for the renormalization of the graph with many loops.

The superficial degree of divergency can be computed easily knowing only the combinatorial datas of each graph. If we denote by

- I the number of internal edges of a given graph,

- $E$ the number of external edges,

- $V$ the number of vertices,

- $L$ the number of loops ( $L=I-V+1$ because of conservation of momentum at each vertex),

then for the Klein-Gordon field we have

$$
\omega(\Gamma)=D L-2 I=D+(D-2) I-D V
$$

where $D$ is the dimension of the base-space. In fact, the transformation $q \rightarrow t q$ gives

$$
\begin{aligned}
\frac{d^{D} q}{(2 \pi)^{D}} & \longrightarrow t^{D} \frac{d^{D} q}{(2 \pi)^{D}}, \\
\frac{1}{q^{2}+m^{2}} & \longrightarrow t^{-2} \frac{1}{q^{2}+m^{2}},
\end{aligned}
$$

therefore to compute $\omega(\Gamma)$ we have to add a term $D$ for each loop, and a term -2 for each internal edge.

In particular, for the $\phi^{3}$-theory (the field $\phi$ with interacting Lagrangian proportional to $\phi^{3}$ ), we have an additional relation $3 V=E+2 I$, and therefore

$$
\omega(\Gamma)=D+\frac{D-6}{2} V-\frac{D-2}{2} E .
$$

9.4 - Regularization: yes or not. Let $\Gamma$ be a divergent graph, that is, we suppose that the amplitude $A(\Gamma)$ presents an ultraviolet divergency. In order to extract the renormalized amplitude $A^{r e n}(\Gamma)$, we can not work directly on $A(\Gamma)$, which is infinite. Instead, there are the following two main possibilities.

Regularization: We can modify $A(\Gamma)$ into a new integral $A_{\rho}(\Gamma)$, called regularized amplitude, by introducing a regularization parameter $\rho$ such that

- $A_{\rho}(\Gamma)$ converges,

- $A_{\rho}(\Gamma)$ reproduces the divergency of $A(\Gamma)$ in a certein limit $\rho \rightarrow \rho_{0}$.

The regularized amplitude $A_{\rho}(\Gamma)$ is then a well-defined function of the external momenta with values which depends on the parameter $\rho$. Let us denote by $R_{\rho}$ the ring of such values.

Then we can modify the function $A_{\rho}(\Gamma)$ into a new function $A_{\rho}^{r e n}(\Gamma)$ such that the limit

$$
A^{r e n}(\Gamma)=\lim _{\rho \rightarrow \rho_{0}} A_{\rho}^{r e n}(\Gamma)
$$

is finite and compatible with the normalization conditions.

Since we are dealing here with ultraviolet divergencies, it suffices to choose as regularization parameter a cut-off $\Lambda \in \mathbb{R}^{+}$which bounds the integrated variables by above. If we denote by $I\left(\Gamma ; q_{1}, \ldots, q_{\ell}\right)$ the integrand of $A(\Gamma)$, that is

$$
A(\Gamma)=\int \frac{d^{D} q_{1}}{(2 \pi)^{D}} \cdots \frac{d^{D} q_{\ell}}{(2 \pi)^{D}} I\left(\Gamma ; q_{1}, \ldots, q_{\ell}\right),
$$

the regularized amplitude can be choosen as

$$
A_{\Lambda}(\Gamma)=\int_{\left|q_{i}\right| \leq \Lambda} \frac{d^{D} q_{1}}{(2 \pi)^{D}} \cdots \frac{d^{D} q_{\ell}}{(2 \pi)^{D}} I\left(\Gamma ; q_{1}, \ldots, q_{\ell}\right),
$$


which reproduces the divergency of $A(\Gamma)$ for $\Lambda \rightarrow \infty$. Alternatively, the regularized amplitude $A_{\Lambda}(\Gamma)$ can also be described as

$$
A_{\Lambda}(\Gamma)=\int \frac{d^{D} q_{1}}{(2 \pi)^{D}} \cdots \frac{d^{D} q_{\ell}}{(2 \pi)^{D}} \chi_{\Lambda}\left(\left|q_{1}\right|, \ldots,\left|q_{\ell}\right|\right) I\left(\Gamma ; q_{1}, \ldots, q_{\ell}\right),
$$

where $\chi_{\Lambda}\left(\left|q_{1}\right|, \ldots,\left|q_{\ell}\right|\right)$ is the step function with value 1 for $\left|q_{1}\right|, \ldots,\left|q_{\ell}\right| \leq \Lambda$ and value 0 for $\left|q_{1}\right|, \ldots,\left|q_{\ell}\right|>\Lambda$.

Beside the cut-off, there exist other possible regularizations. One of the most frequently used is the dimensional regularization, which modifies the real dimension $D$ by a complex parameter $\varepsilon$ such that $A_{\varepsilon}(\Gamma)$ reproduces the divergency of $A(\Gamma)$ for $\varepsilon \rightarrow 0$. Since this regularization demands many explanations, and we are not going to use it here, we omit the details which can be found in [24] or [19].

Integrand functions: The integrand $I\left(\Gamma ; q_{1}, \ldots, q_{\ell}\right)$ of $A(\Gamma)$ is a well defined (rational) function of the variables $q_{1}, \ldots, q_{\ell}$. Therefore we can work directly with the integrand in order to modify it into a new function $I^{r e n}\left(\Gamma ; q_{1}, \ldots, q_{\ell}\right)$, called renormalized integrand, such that

$$
A^{r e n}(\Gamma)=\int \frac{d^{D} q_{1}}{(2 \pi)^{D}} \cdots \frac{d^{D} q_{\ell}}{(2 \pi)^{D}} I^{r e n}\left(\Gamma ; q_{1}, \ldots, q_{\ell}\right)
$$

is finite. This method was used by Bogoliubov in his first formulation of the renormalization, and by Zimmermann in the final prove of the so-called BPHZ formula (cf. 9.9). Its main advantage is that it is independent of the choice of a regularization. For these reasons we adopt it here.

9.5 - Renormalization of a simple loop: Bogoliubov's subtraction scheme. Let $\Gamma$ be a 1PI graph with one loop and superficial degree of divergency $\omega(\Gamma) \geq 0$. We suppose that $\Gamma$ has $k$ external legs with external momentum $p_{1}, \ldots, p_{k}$, then the bare amplitude of the graph is

$$
A\left(\Gamma ; p_{1}, \ldots, p_{k}\right)=\int \frac{d^{D} q}{(2 \pi)^{D}} I\left(\Gamma ; p_{1}, \ldots, p_{k} ; q\right) .
$$

Let $T^{\omega(\Gamma)}$ denote the operator which computes the Taylor expansion in the momentum variables $\mathbf{p}=\left(p_{1}, \ldots, p_{k}\right)$ around the point $\mathbf{p}=0$, up to the degree $\omega(\Gamma)$. Then Bogoliubov and Parasiuk proved in $[1,22]$ (see also [2]) that the integral

$$
A^{r e n}\left(\Gamma ; p_{1}, \ldots, p_{k}\right)=\int \frac{d^{D} q}{(2 \pi)^{D}}\left(I\left(\Gamma ; p_{1}, \ldots, p_{k} ; q\right)-T^{\omega(\Gamma)}\left[I\left(\Gamma ; p_{1}, \ldots, p_{k} ; q\right)\right]\right)
$$

is finite. Changing the value of $\mathbf{p}=0$ to another value $\mathbf{p}=\mathbf{p}_{0}$ amounts to change $A^{\text {ren }}(\Gamma)$ by a finite value. Eventually, the parameter $\mathbf{p}_{0}$ can then be chosen according to the normalization conditions.

By convention, the finite result is expressed as a sum

$$
A^{r e n}\left(\Gamma ; p_{1}, \ldots, p_{k}\right)=A\left(\Gamma ; p_{1}, \ldots, p_{k}\right)+C\left(\Gamma ; p_{1}, \ldots, p_{k}\right),
$$

where the divergent term

$$
C\left(\Gamma ; p_{1}, \ldots, p_{k}\right)=-\int \frac{d^{D} q}{(2 \pi)^{D}} T^{\omega(\Gamma)}\left[I\left(\Gamma ; p_{1}, \ldots, p_{k} ; q\right)\right],
$$

is called the counterterm of the graph $\Gamma$.

9.6 - Locality of counterterms. For a given graph $\Gamma$, the counterterm $C(\Gamma)$ is a function of the external momenta $p_{1}, \ldots, p_{k}$. In fact, as proved by $\mathrm{S}$. Weinberg in [27], it is a polynomial in the variables $p_{i}$ with degree $\omega(\Gamma)$, of the form

$$
C\left(\Gamma ; p_{1}, \ldots, p_{k}\right)=-\left.\int \frac{d^{D} q}{(2 \pi)^{D}} I(\Gamma)\right|_{\mathbf{p}=0}-\left.\sum_{i, \mu} p_{i}^{\mu} \int \frac{d^{D} q}{(2 \pi)^{D}} \frac{\partial I(\Gamma)}{\partial p_{i}^{\mu}}\right|_{\mathbf{p}=0}-\left.\frac{1}{2} \sum_{\substack{i, j \\ \mu, \nu}} p_{i}^{\mu} p_{j}^{\nu} \int \frac{d^{D} q}{(2 \pi)^{D}} \frac{\partial^{2} I(\Gamma)}{\partial p_{i}^{\mu} \partial p_{j}^{\nu}}\right|_{\mathbf{p}=0}-\ldots
$$


This property is called the locality of the counterterms. In matrix notations, with $\mathbf{p}=\left(p_{1}, \ldots, p_{k}\right)$, we can write

$$
C(\Gamma ; \mathbf{p})=C_{0}(\Gamma)+C_{1}(\Gamma) \mathbf{p}+C_{2}(\Gamma) \mathbf{p}^{2}+\cdots+C_{\omega(\Gamma)}(\Gamma) \mathbf{p}^{\omega(\Gamma)},
$$

where the coefficients

$$
C_{r}(\Gamma)=-\left.\frac{1}{r !} \int \frac{d^{D} q}{(2 \pi)^{D}} \partial_{\mathbf{p}}^{r} I(\Gamma)\right|_{\mathbf{p}=0}
$$

involve the derivatives of the integrand at $\mathbf{p}=0$, and are usually directly related to the normalization conditions. Therefore, the finiteness of the number of coefficients in the polynomials describing all the countertems is equivalent to the renormalisability of the theory.

\section{7 - Examples: renormalization of a simple loop.}

a) Let us consider the graph $\Gamma=P^{p}$, in dimension $D=4$. Its amplitude is

$$
A(\Gamma ; p)=\int \frac{d^{4} q}{(2 \pi)^{4}} \frac{1}{q^{2}+m^{2}} \frac{1}{(p-q)^{2}+m^{2}} .
$$

Since $E=2$ and $V=2$ we have $\omega(\Gamma)=0$, therefore the graph $\Gamma$ has a logarithmic divergency. According to the subtraction scheme, its renormalized amplitude is $A^{r e n}(\Gamma ; p)=A(\Gamma ; p)+C(\Gamma)$, where the counterterm

$$
C(\Gamma)=-\left.\int \frac{d^{4} q}{(2 \pi)^{4}} I(\Gamma)\right|_{p=0}=-\int \frac{d^{4} q}{(2 \pi)^{4}} \frac{1}{\left(q^{2}+m^{2}\right)^{2}}
$$

does not dependent on $p$. The integral $A^{r e n}(\Gamma ; p)$ is indeed finite, because

$$
I(\Gamma)-\left.I(\Gamma)\right|_{p=0}=\frac{1}{\left(q^{2}+m^{2}\right)^{2}} \frac{2 p q-p^{2}}{(p-q)^{2}+m^{2}}
$$

behaves like $\frac{1}{|q|^{5}}$ for $|q| \rightarrow \infty$, and therefore $A^{r e n}(\Gamma ; p)=\int \frac{d^{4} q}{(2 \pi)^{4}}\left(I(\Gamma)-\left.I(\Gamma)\right|_{p=0}\right)$ behaves like

$$
\int_{|q|_{\text {min }}}^{\infty} \frac{d^{4}|q|}{|q|^{5}} \simeq \int_{|q|_{\text {min }}}^{\infty} \frac{d|q|}{|q|^{5-3}}=\left[-\frac{1}{|q|}\right]_{|q|_{\text {min }}}^{\infty}=\frac{1}{|q|_{\text {min }}}
$$

b) Let us consider the same graph $\Gamma=\overbrace{}^{p}$, but in dimension $D=6$. Its amplitude is

$$
A(\Gamma ; p)=\int \frac{d^{6} q}{(2 \pi)^{6}} \frac{1}{q^{2}+m^{2}} \frac{1}{(p-q)^{2}+m^{2}} .
$$

Since $E=2$ and $V=2$ we have $\omega(\Gamma)=2$, therefore the graph $\Gamma$ has a quadratic divergency. Then $A^{\text {ren }}(\Gamma ; p)=$ $A(\Gamma ; p)+C(\Gamma ; p)$, where the counterterm is

$$
C(\Gamma ; p)=-\int \frac{d^{6} q}{(2 \pi)^{6}} T^{2}[I(\Gamma)]=C_{0}(\Gamma)+p C_{1}(\Gamma)+p^{2} C_{2}(\Gamma)
$$

with

$$
\begin{aligned}
& C_{0}(\Gamma)=-\int \frac{d^{6} q}{(2 \pi)^{6}} \frac{1}{\left(q^{2}+m^{2}\right)^{2}}, \\
& C_{1}(\Gamma)=-\int \frac{d^{6} q}{(2 \pi)^{6}} \frac{2 q}{\left(q^{2}+m^{2}\right)^{3}}=0 \quad \text { (because the integrand is an odd function), } \\
& C_{2}(\Gamma)=-\int \frac{d^{6} q}{(2 \pi)^{6}} \frac{3 q^{2}-m^{2}}{\left(q^{2}+m^{2}\right)^{4}} .
\end{aligned}
$$


Since the function

$$
I(\Gamma)-T^{2}[I(\Gamma)]=\frac{4 p^{3} q^{3}-3 p^{4} q^{2}-4 m^{2} p^{3} q+m^{2} p^{4}}{\left(q^{2}+m^{2}\right)^{4}\left[(p-q)^{2}+m^{2}\right]} .
$$

has leading term of order $\frac{|q|^{3}}{|q|^{10}}=\frac{1}{|q|^{7}}$ for $|q| \rightarrow \infty$, its integral $A^{\text {ren }}(\Gamma ; p)$ behaves like

$$
\int_{|q|_{\text {min }}}^{\infty} \frac{d^{6}|q|}{|q|^{7}} \simeq \int_{|q|_{\text {min }}}^{\infty} \frac{d|q|}{|q|^{7-5}}=\left[-\frac{1}{|q|}\right]_{|q|_{\text {min }}}^{\infty}=\frac{1}{|q|_{\text {min }}},
$$

and therefore it converges.

Exercice: Check that the counterterm $C_{0}(\Gamma)$ is not sufficient to make the amplitude converging.

c) Let us consider the graph $\Gamma=p_{1} \rightarrow p_{p_{1}-p_{2}}^{p_{2}}$ in dimension $D=6$. Its amplitude is

$$
A\left(\Gamma ; p_{1}, p_{2}\right)=\int \frac{d^{6} q}{(2 \pi)^{6}} \frac{1}{q^{2}+m^{2}} \frac{1}{\left(q+p_{2}\right)^{2}+m^{2}} \frac{1}{\left(q-p_{1}\right)^{2}+m^{2}} .
$$

Since $E=3$ and $V=3$ we have $\omega(\Gamma)=0$, therefore $\Gamma$ has a logarithmic divergency. Then the renormalized amplitude is $A^{r e n}\left(\Gamma ; p_{1}, p_{2}\right)=A\left(\Gamma ; p_{1}, p_{2}\right)+C(\Gamma)$, where

$$
C(\Gamma)=-\left.\int \frac{d^{6} q}{(2 \pi)^{6}} I\left(\Gamma ; p_{1}, p_{2} ; q\right)\right|_{p_{i}=0}=-\int \frac{d^{6} q}{(2 \pi)^{6}} \frac{1}{\left(q^{2}+m^{2}\right)^{3}} .
$$

In fact, the function

$$
\begin{aligned}
I(\Gamma)-\left.I(\Gamma)\right|_{p_{i}=0} & =\frac{1}{q^{2}+m^{2}}\left(\frac{1}{\left[\left(q-p_{1}\right)^{2}+m^{2}\right]\left[\left(q+p_{2}\right)^{2}+m^{2}\right]}-\frac{1}{\left(q^{2}+m^{2}\right)^{2}}\right) \\
& =\frac{2\left(p_{1}-p_{2}\right) q^{3}-\left(p_{1}^{2}-4 p_{1} p_{2}+p_{2}^{2}\right) q^{2}-2\left[p_{1} p_{2}\left(p_{1}-p_{2}\right)+m^{2}\left(p_{1}+p_{2}\right)\right] q-\left(p_{1}^{2} p_{2}^{2}+m^{2} p_{1}+m^{2} p_{2}^{2}\right)}{\left(q^{2}+m^{2}\right)^{3}\left[\left(q-p_{1}\right)^{2}+m^{2}\right]\left[\left(q+p_{2}\right)^{2}+m^{2}\right]}
\end{aligned}
$$

has leading term $\frac{|q|^{3}}{|q|^{10}}=\frac{1}{|q|^{7}}$, and therefore its integral in dimension 6 converges, as in example b).

9.8 - Divergent subgraphs. The subtraction scheme employed for graphs with one loop does not work for graphs with many loops, because of the possible presence of divergent subgraphs.

For instance, consider the graph

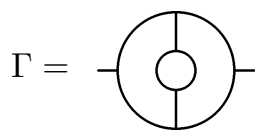

in dimension $D=4$. Since $E=2$ and $V=6$, the graph has negative superficial degree of divergency $\omega(\Gamma)=-4$. According to the subtraction's scheme, then, it should have a zero counterterm $C(\Gamma)$. However, the graph $\Gamma$ contains the 1PI subgraph $\gamma=-\bigcirc$ which has $\omega(\gamma)=0$ in dimension $D=4$ (as we computed in the first of Examples 9.7). Since $\gamma$ diverges, the graph $\Gamma$ diverges too, even if $\omega(\Gamma)$ is strictly negative.

9.9 - Renormalization of many loops: BPHZ algorithm. Let $\Gamma$ be a 1PI graph with many loops and superficial degree of divergency $\omega(\Gamma) \geq 0$, and/or containing some divergent subgraphs. Let $A\left(\Gamma ; p_{1}, \ldots, p_{k}\right)$ be its amplitude and $I(\Gamma) \equiv I\left(\Gamma ; p_{1}, \ldots, p_{k} ; q_{1}, \ldots, q_{\ell}\right)$ its integrand, where $\ell$ is the number of loops of $\Gamma$.

Then, the BPHZ Formula, also called Forest Formula, states that the renormalized (i.e. finite) amplitude of $\Gamma$ is given by

$$
A^{r e n}\left(\Gamma ; p_{1}, \ldots, p_{k}\right)=\int \frac{d^{D} q_{1}}{(2 \pi)^{D}} \cdots \frac{d^{D} q_{\ell}}{(2 \pi)^{D}}\left(I^{\text {prep }}(\Gamma)-T^{\omega(\Gamma)}\left[I^{\text {prep }}(\Gamma)\right]\right),
$$


where $I^{\text {prep }}(\Gamma)$ denotes a prepared term where all the divergent subgraphs have been renormalized. The prepared term is defined recursively on the 1PI divergent subgraphs of $\Gamma$, as follows:

$$
I^{\text {prep }}(\Gamma)=I(\Gamma)+\sum_{\gamma_{i}} \prod_{i}\left(-T^{\omega\left(\gamma_{i}\right)}\left[I^{\text {prep }}\left(\gamma_{i}\right)\right]\right) I\left(\Gamma /\left\{\gamma_{i}\right\}\right),
$$

where the sum is over all 1PI divergent proper subgraphs $\gamma_{i}$ of $\Gamma$ (that is, the subgraphs different from $\Gamma$ itself), such that $\gamma_{i} \cap \gamma_{j}=\emptyset$ (that is, they are disjoint). The notation $\Gamma /\left\{\gamma_{i}\right\}$ means the graph obtained by sqeezing the graphs $\gamma_{i}$ to a point. We explain the details on some examples. The proof was first partially given by Bogoliubov and Parasiuk in 1957 [1], then ameliorated by Hepp in 1966 [16] and finally established by Zimmermann in 1969 $[28]$.

Note that if we extend the definition (9.5.2) of the counterterm to a graph with many loops as follows:

$$
C\left(\Gamma ; p_{1}, \ldots, p_{k}\right)=-\int \frac{d^{D} q_{1}}{(2 \pi)^{D}} \cdots \frac{d^{D} q_{\ell}}{(2 \pi)^{D}} T^{\omega(\Gamma)}\left[I^{p r e p}\left(\Gamma ; p_{1}, \ldots, p_{k} ; q_{1}, \ldots, q_{\ell}\right)\right],
$$

that is, we apply the Taylor expansion to the prepared integrand $I^{p r e p}(\Gamma)$ instead of the bare integrand $I(\Gamma)$, then the renormalized amplitude given by Eq. (9.9.1) can be written as

$$
A^{r e n}\left(\Gamma ; p_{1}, \ldots, p_{k}\right)=A(\Gamma)+C(\Gamma)+\sum_{\gamma_{i}} \prod_{i} C\left(\gamma_{i}\right) A\left(\Gamma /\left\{\gamma_{i}\right\}\right)
$$

Similarly, we can also rewrite the counterterm given by Eq. (9.9.3) as

$$
C\left(\Gamma ; p_{1}, \ldots, p_{k}\right)=-T^{\omega(\Gamma)}\left[A(\Gamma)+\sum_{\gamma_{i}} \prod_{i} C\left(\gamma_{i}\right) A\left(\Gamma /\left\{\gamma_{i}\right\}\right)\right] .
$$

\subsection{0 - Examples: renormalization of many loops.}

a) Let us consider the graph $\Gamma=p_{1} \longrightarrow p_{1}-p_{2}$

$$
\begin{aligned}
A\left(\Gamma ; p_{1}, p_{2}\right)=\int \frac{d^{6} q_{1}}{(2 \pi)^{6}} \frac{d^{6} q_{2}}{(2 \pi)^{6}} \frac{1}{q_{1}^{2}+m^{2}} \frac{1}{\left(p_{1}-q_{1}\right)^{2}+m^{2}} \frac{1}{\left(q_{1}-q_{2}\right)^{2}+m^{2}} \\
\quad \times \frac{1}{q_{2}^{2}+m^{2}} \frac{1}{\left(p_{1}-q_{2}\right)^{2}+m^{2}} \frac{1}{\left(q_{2}-p_{2}\right)^{2}+m^{2}} .
\end{aligned}
$$

Since $E=3$ and $V=5$ we have $\omega(\Gamma)=0$, therefore the graph $\Gamma$ has a logarithmic superficial divergency. Beside this, the graph $\Gamma$ has two 1PI subgraphs:

- the graph $\gamma=p_{1} \longrightarrow T^{q_{2}}$ has a logarithmic divergency;

- the graph $\gamma^{\prime}={ }^{q_{1}}{ }^{p_{2}}$ has $E=4$ and $V=4$, therefore $\omega\left(\gamma^{\prime}\right)=-2$ : it converges.

In conclusion, $\Gamma$ has one divergent 1PI subgraph, $\gamma$. According to the BPHZ formula (9.9.4), the renormalized amplitude of $\Gamma$ is

$$
A^{r e n}\left(\Gamma ; p_{1}, p_{2}\right)=A\left(\Gamma ; p_{1}, p_{2}\right)+C(\Gamma)+C(\gamma) A\left(\Gamma / \gamma ; p_{1}, p_{2}\right)
$$

where

$$
C(\gamma)=-\left.\int \frac{d^{6} q_{1}}{(2 \pi)^{6}} I\left(\gamma ; p_{1}, q_{2} ; q_{1}\right)\right|_{p_{1}, q_{2}=0}=-\int \frac{d^{6} q_{1}}{(2 \pi)^{6}} \frac{1}{\left(q_{1}^{2}+m^{2}\right)^{3}},
$$


the graph $\Gamma / \gamma=p_{1}{\stackrel{q_{2}}{\longrightarrow}}^{p_{2}}$ has amplitude

$$
A\left(\Gamma / \gamma ; p_{1}, p_{2}\right)=\int \frac{d^{6} q_{2}}{(2 \pi)^{6}} \frac{1}{q_{2}^{2}+m^{2}} \frac{1}{\left(p_{1}-q_{2}\right)^{2}+m^{2}} \frac{1}{\left(q_{2}-p_{2}\right)^{2}+m^{2}},
$$

and the overall counterterm is then

$$
\begin{aligned}
C(\Gamma) & =-\left.\int \frac{d^{6} q_{1}}{(2 \pi)^{6}} \frac{d^{6} q_{2}}{(2 \pi)^{6}}\left(I\left(\Gamma ; p_{1}, p_{2} ; q_{1}, q_{2}\right)-\left.I\left(\gamma ; p_{1}, q_{2} ; q_{1}\right)\right|_{p_{1}, q_{2}=0} I\left(\Gamma / \gamma ; p_{1}, p_{2} ; q_{2}\right)\right)\right|_{p_{1}, p_{2}=0} \\
& =-\int \frac{d^{6} q_{1}}{(2 \pi)^{6}} \frac{d^{6} q_{2}}{(2 \pi)^{6}}\left(\frac{1}{\left(q_{1}^{2}+m^{2}\right)^{2}} \frac{1}{\left(q_{1}-q_{2}\right)^{2}+m^{2}} \frac{1}{\left(q_{2}^{2}+m^{2}\right)^{3}}-\frac{1}{\left(q_{1}^{2}+m^{2}\right)^{3}} \frac{1}{\left(q_{2}^{2}+m^{2}\right)^{3}}\right) .
\end{aligned}
$$

b) Let us consider the graph $\Gamma=p$

$$
\begin{aligned}
A(\Gamma ; p)=\int \frac{d^{6} q_{1}}{(2 \pi)^{6}} \frac{d^{6} q_{2}}{(2 \pi)^{6}} \frac{d^{6} q_{3}}{(2 \pi)^{6}} \frac{1}{\left(q_{1}^{2}+m^{2}\right)^{2}} \frac{1}{q_{2}^{2}+m^{2}} \frac{1}{\left(q_{1}-q_{2}\right)^{2}+m^{2}} \\
\times \frac{1}{\left(\left(p-q_{1}\right)^{2}+m^{2}\right)^{2}} \frac{1}{q_{3}^{2}+m^{2}} \frac{1}{\left(p-q_{1}-q_{3}\right)^{2}+m^{2}} .
\end{aligned}
$$

Since $E=2$ and $V=6$ we have $\omega(\Gamma)=2$, therefore the graph $\Gamma$ has a quadratic superficial divergency. Moreover, the graph $\Gamma$ has two 1PI subgraphs, $\gamma_{1}=\gamma_{2}=-\bigcirc$, which have a quadratic divergency. Therefore, according to the BPHZ formula, the renormalized amplitude of $\Gamma$ can be found as follows:

$$
A^{r e n}(\Gamma)=A(\Gamma)+C(\Gamma)+C\left(\gamma_{1}\right) A\left(\Gamma / \gamma_{1}\right)+C\left(\gamma_{2}\right) A\left(\Gamma / \gamma_{2}\right)+C\left(\gamma_{1}\right) C\left(\gamma_{2}\right) A\left(\Gamma / \gamma_{1} \gamma_{2}\right),
$$

where $\Gamma / \gamma_{1}=\Gamma / \gamma_{2}=-9$, and $\Gamma / \gamma_{1} \gamma_{2}=-\bigcirc$. Note that, this time, all the counterterms depend explicitely on the external momenta of the graphs and of the subgraphs. We leave as an exercise the task of writing down their expression.

Finally note that if we call $\gamma$ the graphs $\gamma_{1}=\gamma_{2}$, and we omit the dependence on the external momenta, the renormalized amplitude can be written as

$$
A^{r e n}(\Gamma)=A(\Gamma)+C(\Gamma)+2 C(\gamma) A(\Gamma / \gamma)+C(\gamma)^{2} A\left(\Gamma / \gamma^{2}\right) .
$$

\section{Dyson's renormalization formulas for Green's functions}

As we fixed in Section 5, the aim of quantum field theory is to compute the full Green's functions $\left\langle\phi\left(x_{1}\right) \cdots \phi\left(x_{k}\right)\right\rangle$. To do this, we need to compute the connected Green's functions $G\left(x_{1}, \ldots, x_{k}\right)$, which can only be found perturbatively, as formal series in the powers of the coupling constant $\lambda$. In Lecture III we showed that the coefficients of these series can be labelled by suitable Feynman graphs. Therefore the connected Green's functions can be written as

$$
G\left(x_{1}, \ldots, x_{k}\right)=\sum_{n=0}^{\infty} \lambda^{n} \sum_{V(\Gamma)=n} \frac{\hbar^{L(\Gamma)}}{\operatorname{Sym}(\Gamma)} A\left(\Gamma ; x_{1}, \ldots, x_{k}\right),
$$

where the sum is over all the connected Feynman graphs with $k$ external legs. In Section 9, then, we pointed out the problem of divergencies, which affects some graphs with loops, and showed how to extract a finite contribution for each graph, the renormalized amplitude. Summing up all the renormalized amplitudes, we obtain the renormalized connected Green's functions

$$
G^{r e n}\left(x_{1}, \ldots, x_{k}\right)=\sum_{n=0}^{\infty} \lambda^{n} \sum_{V(\Gamma)=n} \frac{\hbar^{L(\Gamma)}}{\operatorname{Sym}(\Gamma)} A^{r e n}\left(\Gamma ; x_{1}, \ldots, x_{k}\right),
$$

and finally the searched renormalized full Green's functions $\left\langle\phi\left(x_{1}\right) \cdots \phi\left(x_{k}\right)\right\rangle^{r e n}$.

In this section, we discuss the direct way from the bare correlation functions $G\left(x_{1}, \ldots, x_{k}\right)$ to the renormalized ones, $G^{r e n}\left(x_{1}, \ldots, x_{k}\right)$, without making use of Feynman graphs. 
10.1 - Bare and renormalized Lagrangian. From the BPHZ formula (9.9.4), it is clear that the passage from the bare to the renormalized amplitudes amounts to adding many terms which contain the counterterms of the divergent subgraphs,

$$
A^{\text {ren }}(\Gamma)=A(\Gamma)+\text { terms. }
$$

Inserting these terms in the connected Green's functions, then, amounts to adding a series in $\lambda$,

$$
G^{r e n}\left(x_{1}, \ldots, x_{k} ; \lambda\right)=G\left(x_{1}, \ldots, x_{k} ; \lambda\right)+\operatorname{series}(\lambda) .
$$

Since the connected Green's functions are completely determined from the Lagrangian $\mathcal{L}(\phi)$, as we saw in Section 5 , the new terms added to $G\left(x_{1}, \ldots, x_{k} ; \lambda\right)$ must correspond to new terms added to $\mathcal{L}(\phi)$,

$$
\mathcal{L}^{r e n}(\phi, \lambda)=\mathcal{L}(\phi, \lambda)+\Delta \mathcal{L}(\phi, \lambda) .
$$

This Lagrangian is called renormalized, in contraposition with the original Lagrangian $\mathcal{L}(\phi, \lambda)$ called bare.

Let us stress that, beside its name, the renormalized Lagrangian has no particular physical meaning: it is only a formal Lagrangian which gives rise to the renormalized (hence physically meaningful) Green's functions, through the standard procedure described in Section 5.

The number of terms appearing in $\Delta \mathcal{L}(\phi)$ tells us if the theory is renormalizable or not: the theory is not renormalizable if the number of terms to be added is infinite.

10.2 - Renormalization factors. If the theory is renormalizable, then $\Delta \mathcal{L}(\phi)$ contains exactly one term proportional to each term of $\mathcal{L}(\phi)$. The factors appearing in each term of the renormalized Lagrangian are called renormalization factors.

To be precise, let us consider again the interacting Klein-Gordon Lagrangian

$$
\mathcal{L}(\phi, m, \lambda)=\frac{1}{2}\left|\partial_{\mu} \phi(x)\right|^{2}+\frac{m^{2}}{2} \phi(x)^{2}-\frac{\lambda}{3 !} \phi(x)^{3},
$$

as a function of the field $\phi$ and of the physical parameters $m$, the mass, and $\lambda$, the coupling constant. Then the terms added by the renormalization can be organized as

$$
\Delta \mathcal{L}(\phi, m, \lambda)=\frac{1}{2} \Delta_{k}(\lambda)\left|\partial_{\mu} \phi(x)\right|^{2}+\frac{m^{2}}{2} \Delta_{m}(\lambda) \phi(x)^{2}-\frac{\lambda}{3 !} \Delta_{\lambda}(\lambda) \phi(x)^{3},
$$

where $\Delta_{k}, \Delta_{m}$ and $\Delta_{\lambda}$ are series in $\lambda$ containing the counterterms of all Feynman graphs. Hence the renormalized Lagrangian is of the form

$$
\begin{aligned}
\mathcal{L}^{r e n}=\mathcal{L}+\Delta \mathcal{L} & =\frac{1}{2}\left|\partial_{\mu} \phi(x)\right|^{2}+\frac{m^{2}}{2} \phi(x)^{2}-\frac{\lambda}{3 !} \phi(x)^{3}+\Delta_{k}(\lambda) \frac{1}{2}\left|\partial_{\mu} \phi(x)\right|^{2}+\Delta_{m}(\lambda) \frac{m^{2}}{2} \phi(x)^{2}-\Delta_{\lambda}(\lambda) \frac{\lambda}{3 !} \phi(x)^{3} \\
& =\frac{1}{2} Z_{3}(\lambda)\left|\partial_{\mu} \phi(x)\right|^{2}+\frac{m^{2}}{2} Z_{m}(\lambda) \phi(x)^{2}-\frac{\lambda}{3 !} Z_{1}(\lambda) \phi(x)^{3},
\end{aligned}
$$

where $Z_{3}(\lambda)=1+\Delta_{k}(\lambda), Z_{m}(\lambda)=1+\Delta_{m}(\lambda)$ and $Z_{1}(\lambda)=1+\Delta_{\lambda}(\lambda)$ are the renormalization factors.

The renormalization factors are completely determined by the counterterms of the divergent graphs. For the $\phi^{3}$ theory in dimension $D=6$, for instance, a graph $\Gamma$ with $E=2$ is quadratically divergent (as we saw in Example $9.7 \mathrm{~b}$ ) and $C(\Gamma ; p)=C_{0}(\Gamma)+p^{2} C_{2}(\Gamma)$ is a polynomial of degree 2 with zero linear term. Instead, a graph $\Gamma$ with $E=3$ is logarithmically divergent (as we saw in Example 9.7 c), therefore $C(\Gamma ; \mathbf{p})=C_{0}(\Gamma)$ is constant. It turns out that in this case the renormalization factors are organized as follows:

$$
\begin{aligned}
& Z_{3}(\lambda)=1-\sum_{E(\Gamma)=2} \frac{C_{2}(\Gamma)}{\operatorname{Sym}(\Gamma)} \lambda^{V(\Gamma)}, \\
& Z_{m}(\lambda)=1-\sum_{E(\Gamma)=2} \frac{C_{0}(\Gamma)}{\operatorname{Sym}(\Gamma)} \lambda^{V(\Gamma)}, \\
& Z_{1}(\lambda)=1+\sum_{E(\Gamma)=3} \frac{C_{0}(\Gamma)}{\operatorname{Sym}(\Gamma)} \lambda^{V(\Gamma)},
\end{aligned}
$$


10.3 - Bare and effective parameters. If we call $\phi_{b}=Z_{3}(\lambda)^{\frac{1}{2}} \phi$, then we have

$$
\mathcal{L}^{r e n}(\phi, m, \lambda)=\frac{1}{2}\left|\partial_{\mu} \phi_{0}(x)\right|^{2}+\frac{m^{2}}{2} Z_{m}(\lambda) Z_{3}(\lambda)^{-1} \phi_{0}(x)^{2}-\frac{\lambda}{3 !} Z_{1}(\lambda) Z(\lambda)^{-\frac{3}{2}} \phi_{0}(x)^{3},
$$

and if we set also

$$
\begin{aligned}
m_{b} & =m Z_{m}(\lambda)^{\frac{1}{2}} Z_{3}(\lambda)^{-\frac{1}{2}}, \\
\lambda_{b} & =\lambda Z_{1}(\lambda) Z_{3}(\lambda)^{-\frac{3}{2}}
\end{aligned}
$$

we finally obtain

$$
\begin{aligned}
\mathcal{L}^{r e n}(\phi, m, \lambda) & =\frac{1}{2}\left|\partial_{\mu} \phi_{b}(x)\right|^{2}+\frac{1}{2} m_{b}^{2} \phi_{b}(x)^{2}-\frac{\lambda_{b}}{3 !} \phi_{b}(x)^{3} \\
& =\mathcal{L}\left(\phi_{b}, m_{b}, \lambda_{b}\right) .
\end{aligned}
$$

In other words, the "formal" Lagrangian in $\phi, m, \lambda$ which produces the "real" (renormalized) Green's functions, is exactely the original Lagrangian, but on "unreal" values of the field, $\phi_{b}$, of the mass, $m_{b}$, and of the coupling constant, $\lambda_{b}$. By definition, the parameters $\phi_{b}, m_{b}, \lambda_{b}$ are formal series in $\lambda$ with coefficients given by the counterterms of the graphs. The are called bare, in contraposition with the physical ones, $\phi, m, \lambda$, which are called effective because they are the measured ones.

10.4 - Dyson's formulas. According to Eq. (10.3.3), the renormalized Lagrangian in the effective parametrs, $\mathcal{L}^{r e n}(\phi, m, \lambda)$, is equal to the bare Lagrangian in the bare parameters, $\mathcal{L}\left(\phi_{b}, m_{b}, \lambda_{b}\right)$. Therefore, the renormalized Green's functions in the effective parameters, $G^{r e n}\left(x_{1}, \ldots, x_{k} ; m, \lambda\right)$, must be related to the bare Green's functions in the bare parameters, $G\left(x_{1}, \ldots, x_{k} ; m_{b}, \lambda_{b}\right)$. This relation is given by the following formula

$$
G^{r e n}\left(p_{1}, \ldots, p_{k} ; m, \lambda\right)=Z_{3}^{-\frac{k}{2}}(\lambda) G\left(p_{1}, \ldots, p_{k} ; m_{b}, \lambda_{b}\right),
$$

where $m_{b}=m_{b}(m, \lambda)$ and $\lambda_{b}=\lambda_{b}(\lambda)$ are the formal series in the powers of $\lambda$ given by Eqs. (10.3.1) and (10.3.2).

In this lecture, this equality is called Dyson's formula, because it was firstly introduced by F. Dyson for quantum electrodynamics in 1949, cf. [10].

10.5 - Renormalization and semidirect product of series. Dyson's formula (10.4.1), together with the formulae (10.3.1) and (10.3.2), answers to the question that we posed at the beginning of this section. Combining all of them, in fact, we get the explicit expression of the renormalized Green's functions from the bare ones, by means of a product and a substitution by suitable formal series in $\lambda$. The transformation from bare to renormalized Green's functions is a semidirect product law.

To show this, let us rewrite Dyson's formula by pointing out only the dependence of the formal series on the parameters $m$ and $\lambda$ :

$$
G^{r e n}(m, \lambda)=Z_{3}^{-\frac{k}{2}}(\lambda) G\left(m_{b}(m, \lambda), \lambda_{b}(\lambda)\right)
$$

In this formula, the quantities

$$
\begin{aligned}
G^{r e n}(m, \lambda) & =G_{0}+\mathcal{O}(\lambda), \\
G\left(m_{b}, \lambda_{b}\right) & =G_{0}+\mathcal{O}\left(\lambda_{b}\right), \\
Z_{3}^{-\frac{k}{2}}(\lambda) & =(1+\mathcal{O}(\lambda))^{-\frac{k}{2}}=1+\mathcal{O}(\lambda)
\end{aligned}
$$

are invertible series in $\lambda$ (with respect to the multiplication, cf. Example 2.8), and the two bare parameters

$$
\begin{aligned}
m_{b} & =m+\mathcal{O}(\lambda), \\
\lambda_{b} & =\lambda+\mathcal{O}\left(\lambda^{2}\right)
\end{aligned}
$$

are formal diffeomorphisms in $\lambda$ (with respect to the substitution or composition, cf. Example 2.9). Therefore Eq. (10.5.1) tells us that the renormalized Green's function can be found as a semidirect product of suitable series in $\lambda$.

The relationship between the renormalization of the Green's functions and the renormalization of each single graph appearing in the perturbative expansions is the main topic of these lectures. It is described in details in the next section. 


\section{Lecture V - Hopf algebra of Feynman graphs and combinatorial groups of renormalization}

In Lecture I we described the Hopf algebra canonically associated to an algebraic or to a proalgebraic group, and gave some examples, for the most common groups. In this lecture, we start from a Hopf algebra on graphs related to the renormalization, and discuss what is the physical meaning of its associated proalgebraic group.

\section{Connes-Kreimer Hopf algebra of Feynaman graphs and diffeographisms}

Following the works $[8,9]$ of A. Connes and D. Kreimer, we choose as a toy model the $\phi^{3}$ theory in dimension $D=6$, for a scalar field $\phi$. Then, the superficial divergency of a Feynman graph $\Gamma$ with $E$ exterior legs is $\omega(\Gamma)=6-2 E$. Therefore, the amplitude $A(\Gamma)$ is superficially divergent only if $\omega(\Gamma) \geq 0$, that is if $E \leq 3$. However we also assume that the 1-point Green function $\langle\phi(x)\rangle$ vanishes. Consequently, we do not consider the tadpole graphs, which have $E=1$ and are superficially divergent.

11.1 - Graded algebra of Feynaman graphs. Let $\mathcal{H}^{\mathrm{CK}}$ be the polynomial algebra over $\mathbb{C}$ generated by the 1PI Feynman graphs of the $\phi^{3}$ theory, with 2 or 3 external legs. The free commutative multiplication between graphs is denoted by the concatenation, and the formal unit is denoted by 1 .

On the algebra $\mathcal{H}^{\mathrm{CK}}$ we consider the grading induced by the number $L$ of loops of the Feynman graphs: the degree of a monomial $\Gamma_{1} \cdots \Gamma_{s}$ in $\mathcal{H}^{\mathrm{CK}}$ is given by $L\left(\Gamma_{1}\right)+\cdots+L\left(\Gamma_{s}\right)$. Then in degree 0 we have only the scalars (multiples of the unit 1 ), and therefore $\mathcal{H}^{\mathrm{CK}}$ is a connected graded algebra. In degree 1 we have only linear combinations of the 1-loop graphs $-\mathrm{O}_{-}$and $\longrightarrow$. In degree 2 we have linear combinations of products of two 1-loop graphs and graphs with 2 loops, and so on for all higher degrees.

The number of vertices $V$ of Feynman graphs can be used as an alternative grading of $\mathcal{H}^{\mathrm{CK}}$. Note, however, that it is not equivalent to the grading by $L$. In fact, according to paragraph 9.3 , if $E$ is the number of external legs of a $\phi^{3}$-graph in $D=6$, then the number of its vertices is $V=2 L+E-2$. Then, at a given degree $L$ by loops, the degree by vertices is $V=2 L$ for graphs with 2 external legs, and $V=2 L+1$ for graphs with 3 external legs. Therefore the grading induced by $V$ is finer then that induced by $L$.

11.2 - Hopf algebra of Feynaman graphs. On the graded algebra $\mathcal{H}^{\mathrm{CK}}$ we consider the coproduct $\Delta$ : $\mathcal{H}^{\mathrm{CK}} \longrightarrow \mathcal{H}^{\mathrm{CK}} \otimes \mathcal{H}^{\mathrm{CK}}$ defined as the multiplicative and unital map given on a generator $\Gamma$ by

$$
\Delta(\Gamma)=\Gamma \otimes 1+1 \otimes \Gamma+\sum_{\gamma_{1}, \ldots, \gamma_{s}} \Gamma / \gamma_{1} \ldots \gamma_{s} \otimes \gamma_{1} \cdots \gamma_{s}
$$

where the sum is over any possible choice of $1 \mathrm{PI}$ proper and disjoint subgraphs $\gamma_{1}, \ldots, \gamma_{s}$ of $\Gamma$. In this formula, the term $\Gamma / \gamma_{1} \ldots \gamma_{s}$ is the graph obtained from $\Gamma$ by replacing each subgraph $\gamma_{i}$ with 2 external legs with a free propagator - , and each subgraph $\gamma_{j}$ with 3 external legs with a vertex graphs $<$. The term $\gamma_{1} \cdots \gamma_{s}$ is a monomial in $\mathcal{H}^{\mathrm{CK}}$, that is a product of graphs.

On $\mathcal{H}^{\mathrm{CK}}$ we also consider the counit $\varepsilon: \mathcal{H}^{\mathrm{CK}} \longrightarrow \mathbb{C}$ defined as the multiplicative and unital map which annihilates the generators, that is such that $\varepsilon(1)=1$ and $\varepsilon(\Gamma)=0$.

The coproduct and the counit so defined are graded algebra maps. Since the algebra $\mathcal{H}^{\mathrm{CK}}$ is connected, we can use the 5-terms equality of paragraph 1.4 to define recursively the antipode $S: \mathcal{H}^{\mathrm{CK}} \longrightarrow \mathcal{H}^{\mathrm{CK}}$. Explicitely, it is the multiplicative and unital map defined on the generators as

$$
S(\Gamma)=-\Gamma-\sum_{\gamma_{1}, \ldots, \gamma_{s}} \Gamma / \gamma_{1} \ldots \gamma_{s} S\left(\gamma_{1}\right) \cdots S\left(\gamma_{s}\right)
$$

In [8], A. Connes and D. Kreimer showed that $\mathcal{H}^{\mathrm{CK}}$ is a commutative and connected graded Hopf algebra, that is, the coproduct, the counit and the antipode satisfy all the compatibility properties listed in Section 1.

11.3 - Group of diffeographisms and renormalization. The Hopf algebra $\mathcal{H}^{\mathrm{CK}}$ is commutative but of course it is not finitely generated. Then, according to the paragraph $3.6, \mathcal{H}^{\mathrm{CK}}$ defines a pro-algebraic group: for any associative and commutative algebra $A$, the set $G^{\mathrm{CK}}(A)$ of $A$-valued characters on $\mathcal{H}^{\mathrm{CK}}$ is a group with the convolution product $\alpha \star \beta=m_{A} \circ(\alpha \otimes \beta) \circ \Delta$. 
Connes and Kreimer showed in [8] that if $\mathcal{A}_{\rho}$ is the algebra of regularized amplitudes for the $\phi^{3}$ theory in dimension $D=6$, then the BPHZ renormalization recursion takes place in the so-called diffeographisms group

$$
G^{\mathrm{CK}}\left(\mathcal{A}_{\rho}\right)=\operatorname{Hom}_{A l g}\left(\mathcal{H}^{\mathrm{CK}}, \mathcal{A}_{\rho}\right) .
$$

More precisely, this means that the bare amplitude map $A$, the regularized amplitude map $A^{\text {ren }}$ and the counterterm map $C$ are characters $\mathcal{H}^{\mathrm{CK}} \longrightarrow \mathcal{A}_{\rho}$, and moreover that the BPHZ renormalization Formula (9.9.4) is equivalent to

$$
A^{r e n}=A \star C .
$$

In fact, for a given 1PI $\phi^{3}$-graph $\Gamma$, Eq. (11.3.2) means that

$$
(A \star C)(\Gamma)=A(\Gamma) C(1)+A(1) C(\Gamma)+\sum_{\gamma_{1}, \ldots, \gamma_{s}} A\left(\Gamma / \gamma_{1} \cdots \gamma_{s}\right) C\left(\gamma_{1} \cdots \gamma_{s}\right)=A^{r e n}(\Gamma),
$$

then, comparing the BPHZ Formula (9.9.4) with the expression (11.2.1) of the coproduct in $\mathcal{H}^{\mathrm{CK}}$, we see that Eq. (11.3.2) is trivially verifyed provided that the counterterm map $C$ is indeed an algebra homomorphism, and therefore $C\left(\gamma_{1} \cdots \gamma_{s}\right)=C\left(\gamma_{1}\right) \cdots C\left(\gamma_{s}\right)$. This fact is due to a peculiar property of the truncated Taylor operator $T^{\omega(\Gamma)}$ which appears in the counterterm of any graph $\Gamma$, as expressed in Formula (9.9.3). Namely, if we denote by $T$ the truncated Taylor expansion, then for any functions $f$ and $g$ of the external momenta we have

$$
T[f g]+T[f] T[g]=T[T[f] g+f T[g]] .
$$

An operator having this property is called a Rota-Baxter operator. The relationship between Rota-Baxter operators and renormalization has been largely investigated by K. Ebrahimi-Fard and L. Guo, see for instance [11].

11.4 - Diffeographisms and diffeomorphisms. In [9], Connes and Kreimer showed that the renormalization of the coupling constant, that is the formula (10.3.2)

$$
\lambda_{b}(\lambda)=\lambda Z_{1}(\lambda) Z_{3}(\lambda)^{-\frac{3}{2}}
$$

defines an inclusion of the coordinate ring of the group of formal diffeomorphisms into the Hopf algebra $\mathcal{H}^{\mathrm{CK}}$.

Let us denote by $\mathcal{H}^{\text {dif }}$ the complex coordinate ring of the proalgebraic group $G^{\text {dif }}$ of formal diffeomorphisms in one variable, as illustrated in paragraph 2.9. Recall that $\mathcal{H}^{\text {dif }}=\mathbb{C}\left[x_{1}, x_{2}, \ldots\right]$ is an infinitely generated commutative Hopf algebra with coproduct

$$
\Delta x_{n}=x_{n} \otimes 1+1 \otimes x_{n}+\sum_{m=1}^{n-1} x_{m} \otimes \sum_{\substack{p_{0}+p_{1}+\cdots+p_{m}=n-m \\ p_{0}, \ldots, p_{m} \geq 0}} x_{p_{0}} x_{p_{1}} \cdots x_{p_{m}}
$$

and counit $\varepsilon\left(x_{n}\right)=0$. Then, the inclusion $\mathcal{H}^{\text {dif }} \hookrightarrow \mathcal{H}^{\mathrm{CK}}$ is defined as follows: consider the expansion (10.2.1) of the renormalization factors in terms of the counterterms of the divergent graphs, namely

$$
\begin{aligned}
& Z_{1}(\lambda)=1+\sum_{E(\Gamma)=3} \frac{C_{0}(\Gamma)}{\operatorname{Sym}(\Gamma)} \lambda^{V(\Gamma)}, \\
& Z_{3}(\lambda)=1-\sum_{E(\Gamma)=2} \frac{C_{2}(\Gamma)}{\operatorname{Sym}(\Gamma)} \lambda^{V(\Gamma)},
\end{aligned}
$$

and assign to a generator $x_{n}$ of $\mathcal{H}^{\text {dif }}$ the combination of Feynman graphs appearing in the coefficient of $\lambda^{n+1}$ in the series $\lambda_{b}=\lambda Z_{1}(\lambda) Z_{3}(\lambda)^{-\frac{3}{2}}$. In [9], Connes and Kreimer proved that this map preserves the coproduct, and therefore it is a morphism of Hopf algebras. 
11.5 - Diffeographisms as generalized series. Connes and Kreimer's result summerized above means in particular that the group of diffeographisms $G^{\mathrm{CK}}\left(\mathcal{A}_{\rho}\right)$ is projected onto the group of formal diffeomorphisms $G^{\text {dif }}\left(\mathcal{A}_{\rho}\right)$ in one variable, with coefficients in the algebra of regularized amplitudes. In this context, formal diffeomorphisms are formal series in the powers of the coupling constant $\lambda$, that is, series of the form

$$
f(\lambda)=\sum_{n=0}^{\infty} f_{n} \lambda^{n+1}
$$

endowed with the composition law.

A useful way to understand the map $G^{\mathrm{CK}}\left(\mathcal{A}_{\rho}\right) \longrightarrow G^{\mathrm{dif}}\left(\mathcal{A}_{\rho}\right)$ is to represent the diffeographisms as a generalization of usual series of the form

$$
f(\lambda)=\sum_{\Gamma} f_{\Gamma} \lambda^{\Gamma}
$$

where the sum is over suitable Feynman diagrams $\Gamma$, the coefficients $f_{\Gamma}$ are taken in the algebra $\mathcal{A}_{\rho}$, and the powers $\lambda^{\Gamma}$ are not monomials in a possibly complex variable $\lambda$, but just formal symbols. The projection $\pi: G^{\mathrm{CK}}\left(\mathcal{A}_{\rho}\right) \longrightarrow G^{\mathrm{dif}}\left(\mathcal{A}_{\rho}\right)$ is simply the dual map of the inclusion $\mathcal{H}^{\mathrm{dif}} \longrightarrow \mathcal{H}^{\mathrm{CK}}$, and sends a diffeographism of the form (11.5.1) into the formal diffeomorphism

$$
\pi(f)(\lambda)=\sum_{n=0}^{\infty}\left(\sum_{V(\Gamma)=n+1} f_{\Gamma}\right) \lambda^{n+1} .
$$

In other words, the projection is induced on the series by the map which sends a graph $\Gamma$ to the number $V(\Gamma)$ of its internal vertices.

Series of the form (11.5.1) are unreal, and of course have no physical meaning. Instead, their images (11.5.2) are usual series, and have a physical meaning in the context of perturbative quantum field theory: the coupling constants are exactely series of this form, summed up over suitable sets of Feynman diagrams. Moreover, the Green's functions and the renormalization factors are series of this form modulo a constant term which makes them being invertible series instead of formal diffeomorphisms. In conclusion, the meaning of Connes and Kreimer's results is that the renormalization procedure takes place in the group $G^{\mathrm{CK}}\left(\mathcal{A}_{\rho}\right)$, even if the physical results are read in the group $G^{\mathrm{dif}}\left(\mathcal{A}_{\rho}\right)$.

11.6 - Diffeographisms and Dyson's formulas. According to Section 10, the result of renormalization is described by Dyson's formulas (10.4.1) directly on usual series in the powers of the coupling constant $\lambda$. As we said, this happens in the semidirect product $G^{\mathrm{dif}}\left(\mathcal{A}_{\rho}\right) \ltimes G^{\mathrm{inv}}\left(\mathcal{A}_{\rho}\right)$ of the groups of formal diffeomorphisms by that of invertible series.

However, these formulas require the knowledge of the renormalization factors. According to (10.2.1), these are known through the computations of the counterterms of all Feynman graphs. In other words, the physical results given by Dyson's formulas seem to be the projection of computations which take place in the semidirect product $G^{\mathrm{CK}}\left(\mathcal{A}_{\rho}\right) \ltimes G_{\text {graphs }}^{\mathrm{inv}}\left(\mathcal{A}_{\rho}\right)$, where $G^{\mathrm{CK}}\left(\mathcal{A}_{\rho}\right)$ is the diffeographisms group dual to the Connes-Kreimer Hopf algebra, and $G_{\text {graphs }}^{\text {inv }}\left(\mathcal{A}_{\rho}\right)$ is a suitable lifting of the group of invertible series whose coordinate ring is spanned by Feynman graphs.

This conjecture has been proved for quantum electrodynamics in the sequel of works [4], [5] and [6]. In those works, the Green's functions are expanded over planar binary trees, that is, planar trees with internal vertices of valence 3, which were used by C. Brouder in [3] as intermediate summation terms between integer numbers and Feynman graphs. It has also been proved by W. van Suijlekom in [26] for any gauge theory. For the $\phi^{3}$-theory the work is in progress.

11.7 - Groups of "combinatorial" series. If the diffeographisms are represented as generalized series of the form (11.5.1), the group law dual to the coproduct in $\mathcal{H}^{\mathrm{CK}}$ should be represented as a "composition" among them. This operation has been defined in principle by P. van der Laan in [25], using operads. An operad is the set of all possible operations of a given type that one can do on any algebra of that type. A particular algebra is then a representation of the corresponding operad. For instance, there exists the operad of associative algebras, that of Lie algebras, and many other examples of operads giving rise to corresponding types of algebras. By 
assumption, operads are endowed with an intrinsic operadic composition which allows to perform the operations one after another one in the corresponding algebras, and still get the result of an operation. The group $G^{\text {dif }}$ of formal diffeomorphisms is deeply related to the operad $\mathcal{A} s$ of associative algebras, and in particular the composition of formal series in one variable can be directly related to the operadic composition in $\mathcal{A} s$. Based on this observation, Van der Laan had the idea to realize the "composition" among diffeographisms as the operadic composition of a suitable operad constructed on Feynman graphs. In [25], he indeed defined an operad of all Feynman graphs, but didn't describe explicitely how to restrict the general construction to the particular case of Feynman graphs for a given theory. In particular, the explicit form of the group $G^{\mathrm{CK}}\left(\mathcal{A}_{\rho}\right)$ related to the renormalization of the $\phi^{3}$-theory is not achieved.

A complete description of the generalized series and their composition law is given in [14] for the renormalization of quantum electrodynamics, on the intermediate coordinate rings spanned by planar binary trees. However, trees are combinatorial objets much simplier to handle then Feynman graphs, and the generalization of this construction to diffeographisms is still uncomplete.

Groups of series expanded over other "combinatorial objects", such as rooted (non-planar) trees, also appear in the context of renormalization. Such trees, in fact, can be used to describe the perturbative expansion of Green's functions, and were used by D. Kreimer in [18] to describe the first Hopf algebra of renormalization appearing in the literature. The dual group of tree-expanded series was then used by F. Girelli, T. Krajewski and P. Martinetti in [15], in their study of Wilson's continuous renormalization group.

Furthermore, the series expanded over various "combinatorial objects" make sense not only in the context of the renormalization of a quantum field theory, but already for classical interacting fields. In fact, as we pointed out in Section 6, these fields are described perturbatively as series expanded over trees. Then, any result on usual series which has a physical meaning should be the projection of computations which take place in the corresponding set of "combinatorial series".

Finally, all the Hopf algebras constructed on "combinatorial objects" which appear in physics share some properties which are investigated in various branches of mathematics. On one side, as we already mentioned, these Hopf algebras seem to be deeply related to operads or to some generalization of them, see for instance the works by J.-L. Loday. On the other side they turn out to be related to the various generalizations of the algebras of symmetric functions, see for instance the several works by J. Y. Thibon and coll., or those by M. Aguiar and F. Sottile, and seem related to the so-called combinatorial Hopf algebras.

\section{References}

[1] N. N. Bogoliubov and O. S. Parasiuk, , Acta Math. 97 (1957), 227.

[2] N. N. Bogoliubov and D. V. Shirkov, Introduction to th etheory of quantized fields, Interscience, New York 1959.

[3] C. Brouder, On the trees of quantum fields, Eur. Phys. J. C 12 (2000), 535-549.

[4] C. Brouder and A. Frabetti, Renormalization of QED with planar binary trees, Eur. Phys. J. C 19 (2001), $715-741$.

[5] C. Brouder and A. Frabetti, QED Hopf algebras on planar binary trees, J. Alg. 267 (2003), 298-322.

[6] C. Brouder, A. Frabetti and C. Krattenthaler, Non-commutative Hopf algebra of formal diffeomorphisms, Adv. Math. 200 (2006), 479-524.

[7] A. Connes and D. Kreimer, Hopf Algebras, Renormalization and Noncommutative Geometry. Comm. Math. Phys. 199 (1998) 203-242.

[8] A. Connes and D. Kreimer, Renormalization in quantum field theory and the Riemann-Hilbert problem. I. The Hopf algebra structure of graphs and the main theorem. Comm. Math. Phys. 210 (2000), 249-273.

[9] A. Connes and D. Kreimer, Renormalization in quantum field theory and the Riemann-Hilbert problem. II. The $\beta$-function, diffeomorphisms and the renormalization group. Comm. Math. Phys. 216 (2001), 215-241.

[10] F. J. Dyson, The S matrix in quantum electrodynamics, Phys. Rev. 76 (1949), 1736-55. 
[11] K. Ebrahimi-Fard and L. Guo, Rota-Baxter Algebras in Renormalization of Perturbative Quantum Field Theory, Fields Institute Communications 50 (2007), 47-105.

[12] H. Epstein and V. J. Glaser, The role of locality in perturbation theory, Ann. Poincar Phys. Theor. A 19 (1973), 211.

[13] F. Faà di Bruno, Sullo sviluppo delle funzioni, Ann. Sci. Mat. Fis., Roma 6 (1855), 479-480.

[14] A. Frabetti, Groups of tree-expanded formal series, Journal of Algebra 319 (2008) 377-413.

[15] F. Girelli, T. Krajewski, P. Martinetti, An algebraic Birkhoff decomposition for the continuous renormalization group, J. Math. Phys. 45 (2004) 4679-4697.

[16] K. Hepp, Proof of the Bogoliubov-Parasiuk Theorem on Renormalization, Comm. Math. Phys. 2 (1966), 301-326.

[17] G. Hochschild, La structure des groupes de Lie, Dunod 1968.

[18] D. Kreimer, On the Hopf algebra structure of perturbative quantum field theories, Adv. Theor. Math. Phys.2 (1998), 303-334.

[19] Itzykson and J.-P. Zuber, Quantum Field Theory, McGraw Hill 1980

[20] I. G. Macdonald, Symmetric functions and Hall polynomials, Oxford University Press 1979.

[21] Â. Mestre, R. Oeckl, Combinatorics of n-point functions via Hopf algebra in quantum field theory, J. Math. Phys. 47 (2006) 052301.

[22] O. S. Parasiuk, , Ukrainskii Math. J. 12 (1960), 287.

[23] R. P. Stanley, Enumerative combinatorics, Cambridge University Press 1997.

[24] G. 't Hooft, M. Veltman, Regularization and renormalization of gauge fields, Nucl. Phys. B 44 (1972), 189-213.

[25] P. van der Laan, Operads and the Hopf algebras of renormalization, preprint (2003), http://www.arxiv.org/abs/math-ph/0311013.

[26] W. van Suijlekom, Multiplicative renormalization and Hopf algebras, preprint (2007), arXiv:0707.0555

[27] S. Weinberg, , Phys. Rev. 118 (1960), 838.

[28] W. Zimmermann, Convergence of Bogoliubov's Method of Renormalization in Momentum Space, Comm. Math. Phys. 15 (1969), 208-234. 